\title{
SIMPLY-CONNECTED 4-MANIFOLDS WITH A GIVEN BOUNDARY
}

\author{
STEVEN BOYER
}

\begin{abstract}
Let $M$ be a closed, oriented, connected 3-manifold. For each bilinear, symmetric pairing $\left(\mathbf{Z}^{n}, L\right)$, our goal is to calculate the set $\mathcal{V}_{L}(M)$ of all oriented homeomorphism types of compact, 1-connected, oriented 4manifolds with boundary $M$ and intersection pairing isomorphic to $\left(\mathbf{Z}^{n}, L\right)$.

For each pair $\left(\mathbf{Z}^{n}, L\right)$ which presents $H_{*}(M)$, we construct a double coset space $B_{L}^{t}(M)$ and a function $c_{L}^{t}: \mathcal{V}_{L}(M) \rightarrow B_{L}^{t}(M)$. The set $B_{L}^{t}(M)$ is the quotient of the group of all link-pairing preserving isomorphisms of the torsion subgroup of $H_{1}(M)$ by two naturally occuring subgroups.

When $\left(\mathbf{Z}^{n}, L\right)$ is an even pairing, we construct another double coset space $\hat{B}_{L}(M)$, a function $\hat{c}_{L}: \mathcal{V}_{L}(M) \rightarrow \hat{B}_{L}(M)$ and a projection $p_{2}: \hat{B}_{L}(M) \rightarrow$ $B_{L}^{t}(M)$ such that $p_{2} \cdot \hat{c}_{L}=c_{L}^{t}$.

Our main result states that when $\left(\mathbf{Z}^{n}, L\right)$ is even the function $\hat{c}_{L}$ is injective, as is the function $c_{L}^{t} \times \Delta: \mathcal{V}_{L}(M) \rightarrow B_{L}^{t}(M) \times \mathbf{Z} / 2$ when $\left(\mathbf{Z}^{n}, L\right)$ is odd. Here $\Delta$ is a Kirby-Siebenmann obstruction to smoothing. It follows that the sets $\mathcal{V}_{L}(M)$ are finite and of an order bounded above by a constant depending only on $H_{1}(M)$. We also show that when $H_{1}(M ; \mathbf{Q}) \cong 0$ and $\left(\mathbf{Z}^{n}, L\right)$ is even, $c_{L}^{t}=p_{2} \cdot \hat{c}_{L}$ is injective.

It seems likely that via the functions $c_{L}^{t} \times \Delta$ and $\hat{c}_{L}$, the sets $B_{L}^{t}(M) \times \mathbf{Z} / 2$ and $\hat{B}_{L}(M)$ calculate $\mathcal{V}_{L}(M)$ when $\left(\mathbf{Z}^{n}, L\right)$ is respectively odd and even. We verify this in several cases, most notably when $H_{1}(M)$ is free abelian.

The results above are based on a theorem which gives necessary and sufficient conditions for the existence of a homeomorphism between two 1connected 4-manifolds extending a given homeomorphism of their boundaries.

The theory developed is then applied to show that there is an $m>0$, depending only on $H_{1}(M)$, such that for any self-homeomorphism $f$ of $M$, $f^{m}$ extends to a self-homeomorphism of any 1-connected, compact 4-manifold with boundary $M$.
\end{abstract}

Introduction. In M. Freedman's fundamental paper [Fr], he classified closed, simply-connected, oriented 4-manifolds up to orientation preserving homeomorphism. He showed that modulo the Kirby-Siebenmann invariant, these manifolds are in bijective correspondence through their intersection pairings with the set of unimodular, bilinear, symmetric pairings over $\mathbf{Z}$ (see Theorem (1.5) of [Fr] and Corollary (2.2.3) of $[\mathbf{Q}])$. In this paper we begin a classification of simply connected 4-manifolds with connected boundary. Our results with regard to this problem are based on a theorem which gives necessary and sufficient conditions for the existence of a homeomorphism between 1-connected 4-manifolds extending a given homeomorphism of their boundaries.

Received by the editors September 19, 1985.

1980 Mathematics Subject Classification (1985 Revision). Primary 57N15.

Research supported by an NSERC (Canada) post-doctoral fellowship. 
Throughout this paper, $M$ will denote a fixed closed, oriented, connected 3manifold. For each symmetric bilinear form $L: \mathbf{Z}^{n} \times \mathbf{Z}^{n} \rightarrow \mathbf{Z}$, consider the set $V_{L}(M)$ of all oriented homeomorphism types of compact, 1-connected, oriented 4manifolds with (oriented) boundary $M$ and whose intersection pairing is isomorphic to $\left(\mathbf{Z}^{n}, L\right)$.

In general $\mathcal{V}_{L}(M)$ will be empty unless $\left(\mathbf{Z}^{n}, L\right)$ presents $H_{*}(M)(\S 1)$. When $M$ is a homology 3 -sphere, this occurs if and only if $L$ is unimodular and in the case $L$ is unimodular, one may combine Theorems $\left(1.4^{\prime}\right)$ and $(1.5)$ of $[\mathbf{F r}]$ to show that

$$
\left|\mathcal{V}_{L}(M)\right|= \begin{cases}1, & L \text { is even } \\ 2, & L \text { is odd }\end{cases}
$$

Further, when $L$ is odd the two manifolds are distinguished by their Kirby-Siebenmann invariants.

To describe our results, let $A^{t}(M)$ denote the group of link pairing preserving isomorphisms of $T_{1}(M)$, the torsion subgroup of $H_{1}(M)$. We let $\nvdash_{+}(M)$ denote the group of orientation preserving homeomorphisms of $M$ and $H_{+}^{t}(M)$ the subgroup of $A^{t}(M)$ induced by elements of $\mathscr{H}_{+}(M)$. When $\left(\mathbf{Z}^{n}, L\right)$ presents $H_{*}(M)$, there is another naturally occurring subgroup $A_{L}^{t}(M) \subseteq A^{t}(M)$ and we form the set $B_{L}^{t}(M)$ of double cosets

$$
B_{L}^{t}(M)=H_{+}^{t}(M) \backslash A^{t}(M) / A_{L}^{t}(M) .
$$

Let $V$ be a 4-manifold with boundary and $\Delta(V) \in \mathbf{Z} / 2$ the Kirby-Siebenmann obstruction to extending the product smooth structure on $\partial V \times \mathbf{R}$ across $V \times \mathbf{R}$.

(0.1) THEOREM. Suppose $\left(\mathbf{Z}^{n}, L\right)$ presents $H_{*}(M)$ and $V$ is a 4-manifold representing a class in $\mathcal{V}_{L}(M)$ with $\Lambda:\left(\mathbf{Z}^{n}, L\right) \rightarrow\left(H_{2}(V), \cdot\right)$ an isometry. Then there is a function $c_{L}^{t}: V_{L}(M) \rightarrow B_{L}^{t}(M)$ which associates to the class of $V$ the double coset $H_{+}^{t}(M) \partial(\Lambda)_{1}^{t} A_{L}^{t}(M)$.

When $L$ is an odd pairing, the function $C_{L}^{t}=c_{L}^{t} \times \Delta: V_{L}(M) \rightarrow B_{L}^{t}(M) \times \mathbf{Z} / 2$ is injective.

When $L$ is an even form presenting $H_{*}(M)$, the function $C_{L}^{t}$ may not be a monomorphism. To deal with this situation, we shall consider the quadratic enhancements of the link pairing on $M$ and use these to define a group $\hat{A}(M) \subseteq$ $A(M) \times S(\operatorname{Spin}(M)), A(M)$ being a certain group of equivalences of $H_{*}(M)$ and $S(\operatorname{Spin}(M))$ the symmetric group on the set of spin structures of $M$.

As before there are subgroups $\hat{H}_{+}(M)$ and $\hat{A}_{L}(M)$ of $\hat{A}(M)$ and we shall consider the double coset space

$$
\hat{B}_{L}(M)=\hat{H}_{+}(M) \backslash \hat{A}(M) / \hat{A}_{L}(M) .
$$

There is a natural projection $p_{2}: \hat{B}_{L}(M) \rightarrow B_{L}^{t}(M)$.

Now the presentation $\left(\mathbf{Z}^{n}, L\right)$ of $H_{*}(M)$ determines a set of spin structures, $\operatorname{Spin}_{L}(M)$, which turns out to be an orbit of the action of

$$
I^{1}(M)=\operatorname{image}\left(H^{1}(M) \rightarrow H^{1}(M ; \mathbf{Z} / 2)\right)
$$

on $\operatorname{Spin}(M)$.

For $(\alpha, \pi) \in \hat{A}(M)$, let $\langle\alpha, \pi\rangle$ denotes its equivalence class in $\hat{B}_{L}(M)$. 
(0.2) THEOREM. Suppose $\left(\mathbf{Z}^{n}, L\right)$ is an even pairing presenting $H_{*}(M)$. Then there is an injective function $\hat{c}_{L}: \mathcal{V}_{L}(M) \rightarrow \hat{B}_{L}(M)$ such that $p_{2} \circ \hat{c}_{L}=c_{L}^{t}$. Further, for each $(\alpha, \pi) \in \hat{A}(M)$, there is a subgroup $\mathfrak{H}_{+}(M, \alpha) \subset \mathfrak{H}_{+}(M)$ which acts on the left of $\pi\left(\operatorname{Spin}_{L}(M)\right)$ and for which there is a bijection

$$
\psi_{(\alpha, \pi)}: \pi\left(\operatorname{Spin}_{L}(M)\right) / \not_{+}(M, \alpha) \rightarrow\left(p_{2}\right)^{-1}\left(p_{2}(\langle\alpha, \pi\rangle)\right) .
$$

Now that when $H_{1}(M ; \mathbf{Q}) \cong 0, I^{1}(M) \cong 0$. Thus if $\left(\mathbf{Z}^{n}, L\right)$ is an even presentation of $H_{*}(M),\left|\operatorname{Spin}_{L}(M)\right|=1$. From Theorems (0.1) and (0.2) we immediately deduce,

(0.3) COROLlaRY. If $H_{1}(M ; \mathbf{Q}) \cong 0$, the functions $c_{L}^{t}$ and $C_{L}^{t}$ are injective when $L$ is respectively even and odd.

More generally, the two theorems show,

(0.4) COROLlaRY. For each symmetric, bilinear form $\left(\mathbf{Z}^{n}, L\right)$, the set $\mathcal{V}_{L}(M)$ is finite.

It is natural to ask whether or not the functions $\hat{c}_{L}$ and $C_{L}^{t}$ are bijections. The work of Freedman alluded to earlier shows that this is indeed the case when $M$ is a homology 3-sphere. It seems reasonable to expect this to hold in general and in $\S 5$ we verify it in several cases, most notably when $T_{1}(M)=0$.

(0.5) Proposition. Suppose $\left(\mathbf{Z}^{n}, L\right)$ presents $H_{*}(M)$ where $H_{*}(M)$ is free abelian. Then $C_{L}^{t}$ (respectively $\hat{c}_{L}$ ) is a bijection when $L$ is odd (respectively even). More precisely, if $L$ is odd, the correspondence $\Delta: \mathcal{V}_{L}(M) \rightarrow \mathbf{Z} / 2$ is bijective. If $L$ is even, the correspondence $\psi_{(1,1)}^{-1} \circ \hat{c}_{L}: \mathcal{V}_{L}(M) \rightarrow \operatorname{Spin}(M) / \mathscr{H}_{+}(M)$ is bijective.

Next we ask under what general conditions on $M$ can we be assured that modulo the Kirby-Siebenmann invariant, a compact, 1-connected, oriented 4-manifold with boundary $M$ is determined by its bilinear form. Clearly this is just asking when $\left|B_{L}^{t}(M)\right|=1$ (respectively $\left|\hat{B}_{L}(M)\right|=1$ ) when $L$ is odd (respectively even). It is possible to construct many specific examples of such manifolds. The following result presents a general criterion for when it will occur.

(0.6) Proposition. Suppose $T_{1}(M) \cong \mathbf{Z} / r$ where $r=1,2,4, p^{n}$ or $2 p^{n}$, $p$ an odd prime. Then if $\left(\mathbf{Z}^{n}, L\right)$ presents $H_{*}(M),\left|B_{L}^{t}(M)\right|=1$. When $L$ is even $\hat{B}_{L}(M)=\operatorname{Spin}_{L}(M) / \not_{+}(M)$. In particular, if $\nvdash_{+}(M)$ acts transitively on $\operatorname{Spin}_{L}(M),\left|\hat{B}_{L}(M)\right|=1$.

Our main technical result is a theorem which gives necessary and sufficient conditions for extending certain elements of $\varkappa_{+}(M)$ to homeomorphisms between 1connected 4-manifolds bounded by $M$.

More precisely let $V_{1}$ and $V_{2}$ be compact, 1-connected, oriented 4-manifolds with boundary $M$ and suppose $f \in \mathscr{H}_{+}(M)$.

There are two obstructions to extending $f$ to a homeomorphism $F: V_{1} \rightarrow V_{2}$. The first is to find an isometry $\Lambda:\left(H_{2}\left(V_{1}\right), \cdot\right) \rightarrow\left(H_{2}\left(V_{2}\right), \cdot\right)$ for which the following diagram commutes.

$$
\begin{aligned}
& 0 \rightarrow H_{2}(M) \rightarrow H_{2}\left(V_{1}\right) \rightarrow H_{2}\left(V_{1}, M\right) \rightarrow H_{1}(M) \rightarrow 0 \\
& \begin{array}{llll}
f_{*} \downarrow & \Lambda \downarrow & \Lambda^{*} \uparrow & \downarrow f_{*}
\end{array} \\
& 0 \rightarrow H_{2}(M) \rightarrow H_{2}\left(V_{2}\right) \rightarrow H_{2}\left(V_{2}, M\right) \rightarrow H_{1}(M) \rightarrow 0
\end{aligned}
$$


Here $\Lambda^{*}$ is the adjoint of $\Lambda$ with respect to the identification of $H_{2}\left(V_{i}, M\right)$ with $\operatorname{Hom}\left(H_{2}\left(V_{i}\right), \mathbf{Z}\right)(i=1,2)$ arising from Lefschetz duality.

We shall call such a pair $(f, \Lambda)$ a morphism and denote it symbolically as $(f, \Lambda): V_{1}$ $\rightarrow V_{2}$.

The second obstruction encountered is to realize a given morphism $(f, \Lambda)$ geometrically. That is, to find a homeomorphism $F: V_{1} \rightarrow V_{2}$ such that $(f, \Lambda)=\left(F \mid M, F_{*}\right)$. In this paper we shall concentrate on the second obstruction, though we do make some analysis and comments on the first (see Proposition (1.6) and Remark (1.13)).

(0.7) THEOREM. If $(f, \Lambda): V_{1} \rightarrow V_{2}$ is a morphism, there is an obstruction class $\theta(f, \Lambda) \in I^{1}(M)$ such that for $(f, \Lambda)$ to be realized geometrically, it is necessary and sufficient that $\Delta\left(V_{1}\right) \equiv \Delta\left(V_{2}\right)(\bmod 2)$ and $\theta(f, \Lambda)=0$.

If $\left(H_{2}\left(V_{1}\right), \cdot\right)$ is even, then $\theta(f, \Lambda)=0$ implies $\Delta\left(V_{1}\right) \equiv \Delta\left(V_{2}\right)$.

The next result lists more information concerning the obstructions $\theta(f, \Lambda)$.

(0.8) Proposition. Let $(f, \Lambda): V_{1} \rightarrow V_{2}$ be a given morphism. Then

(i) if $H_{1}(M ; \mathbf{Q}) \cong 0, \theta(f, \Lambda)=0$;

(ii) if $L$ is odd and $y \in I^{1}(M)$ is arbitrary, there is another morphism $\left(f, \Lambda^{\prime}\right): V_{1}$ $\rightarrow V_{2}$ for which $\theta\left(f, \Lambda^{\prime}\right)=y$. In particular, there is a $\Lambda^{\prime}$ with $\theta\left(f, \Lambda^{\prime}\right)=0$;

(iii) if $L$ is even, then $\theta(f, \Lambda)$ depends only upon $f$. Indeed $\theta(f, \Lambda)=0$ if and only if the manifold $V=V_{1} \cup_{f}\left(-V_{2}\right)$ is spin.

(0.9) CoROllary. Given a morphism $(f, \Lambda): V_{1} \rightarrow V_{2}$, where $\Delta\left(V_{1}\right) \equiv \Delta\left(V_{2}\right)$, then $f$ extends to a homeomorphism $F: V_{1} \rightarrow V_{2}$ as long as $H_{1}(M)$ is finite or the intersection pairing on $V_{1}$ is odd. If this pairing is even, $f$ may not extend.

An example of a nongeometrically realizable morphism is given in the discussion following Proposition (4.1).

The following theorem is an application of all the theory developed above.

(0.10) TheOREM. Let $M$ be a closed, connected, oriented 3-manifold. Then there is an integer $m>0$ depending only on $H_{1}(M)$ such that given any $f \in H_{+}(M)$ and any compact, 1-connected 4-manifold $V$ with boundary $M, f^{m}$ extends to a selfhomeomorphism of $V$.

Note that if the mapping class group of $M$ is finite then Theorem $(0.10)$ is obvious. Examples such as $M=S^{1} \times S^{1} \times S^{1}$ show that this is not always the case.

Several words of acknowledgment are due at this point.

A special case of Theorem (0.7) was proved and used in the author's doctoral dissertation (Theorem (4.3) of [B]) written under the supervision of Professor P. J. Kahn at Cornell University. The approach used there and in this paper is based on a method of C. T. C. Wall for constructing $h$-cobordisms between 1-connected 4manifolds [W1]. He works with the case $M=S^{3}$, though his analysis goes through verbatim when $M$ is a homology 3 -sphere. Our contribution is in dealing with a general (oriented) 3-manifold.

Another approach to Theorem (0.7) is through the use of surgery theory. The idea would be to first attempt to extend a given $f \in \not_{+}(M)$ to a homotopy equivalence $\hat{f}: V_{1} \rightarrow V_{2}$ and then to try and replace $\hat{f}$ by a homeomorphism (see the 
proof of Theorem (1.5) of [Fr] for instance). In unpublished work [Mo], John Morgan studied the problem of extending homotopy equivalences across 1-connected 4-manifolds and proved, amongst other things, a homotopy version of Theorem (0.7). Part (ii) of Proposition (0.8) is based on ideas from this work. The author would like to thank R. Kirby who informed him of Morgan's results upon receipt of an earlier version of this paper.

We would also like to point out that in independent work [V], Pierre Vogel has announced certain classification and realization results for bounded 1-connected, 4-dimensional Poincaré complexes and manifolds.

We thank Andrew Nicas for pointing out and discussing the relevance of quadratic enhancements of the link pairing when $\left(\mathbf{Z}^{n}, L\right)$ is even.

Finally we would like to acknowledge the hospitality of both Cambridge University and the University of Toronto during the preparation of this work.

The paper is organized in the following fashion. $\S 1$ provides the algebraic background necessary for the analysis of $\mathcal{V}_{L}(M)$ when $L$ is odd. In $\S \S 2$ and 3 , spin structures on manifolds and the quadratic enhancements of the link pairing on $T_{1}(M)$ are introduced and used to provide the algebraic structure needed when $L$ is even. $\S 4$ contains the proof of Theorem (0.7) and in $\S 5$ the theory previously developed is applied to prove Theorems (0.1) and (0.2).

1. We shall assume throughout that all manifolds are compact, oriented and that

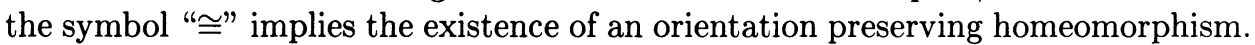
Boundaries of manifolds will have the orientation corresponding to the boundary of the fundamental class of the manifold they bound.

As in the introduction, $M$ will denote a closed, connected 3-manifold and $T_{1}(M)$ the torsion subgroup of $H_{1}(M)$. Any endomorphism of $H_{1}(M), \phi$ say, induces an endomorphism of $T_{1}(M)$ by restriction, which we shall write as $\phi^{t}: T_{1}(M) \rightarrow$ $T_{1}(M)$. The link pairing on $T_{1}(M)$ will be denoted $l_{M}: T_{1}(M) \times T_{1}(M) \rightarrow \mathbf{Q} / \mathbf{Z}$.

A bilinear form space is a pair $\left(\mathbf{Z}^{n}, L\right)$ where $L: \mathbf{Z}^{n} \times \mathbf{Z}^{n} \rightarrow \mathbf{Z}$ is a symmetric bilinear pairing. For instance, if $V$ is a 4-manifold, the intersection pairing $\left(\mathrm{H}_{2}(V), \cdot\right)$ is such a pairing and we shall call these forms geometric.

A form $\left(\mathbf{Z}^{n}, L\right)$ is said to be even if $L(\xi, \xi) \equiv 0(\bmod 2)$ for each $\xi \in \mathbf{Z}^{n}$ and odd otherwise.

An isometry of bilinear form spaces is an isomorphism of the underlying groups which preserves the pairings.

If $A$ is an abelian group, $A^{*}$ will denote the dual group $\operatorname{Hom}(A, \mathbf{Z})$.

(1.1) Definition. A bilinear form space $\left(\mathbf{Z}^{n}, L\right)$ presents $H_{*}(M)$ if there is an exact sequence

$$
0 \rightarrow H_{2}(M) \stackrel{h}{\rightarrow} \mathbf{Z}^{n} \stackrel{\operatorname{ad}(L)}{\rightarrow}\left[\mathbf{Z}^{n}\right]^{*} \stackrel{\partial}{\rightarrow} H_{1}(M) \rightarrow 0
$$

such that

(i) if $\operatorname{ad}(L)\left(\xi_{i}\right)=m_{i} \eta_{i}(i=1,2)$ where $m_{1} m_{2} \neq 0$, then

$$
l_{M}\left(\partial \eta_{1}, \partial \eta_{2}\right) \equiv-\frac{1}{m_{1} m_{2}} L\left(\xi_{1}, \xi_{2}\right)
$$

(ii) if $\beta \in H_{2}(M)$ and $\eta \in\left[\mathbf{Z}^{n}\right]^{*}$, then $\partial(\eta) \cdot \beta=\eta(h(\beta))$.

For instance if $M$ is the boundary of a 4-manifold $V$, then it is well known that $\left(H_{2}(V), \cdot\right)$ presents $H_{*}(M)$ (see $\S 3$ of $\left.[\mathbf{G}, \mathbf{L}]\right)$. 
(1.2) Definition. An automorphism of $H_{*}(M)$ is a pair of isomorphisms $\alpha=\left(\alpha_{1}, \alpha_{2}\right), \alpha_{i}: H_{i}(M) \rightarrow H_{i}(M)(i=1,2)$, such that

(i) if $\nu_{1}, \nu_{2} \in T_{1}(M)$, then $l_{M}\left(\alpha_{1}\left(\nu_{1}\right), \alpha_{1}\left(\nu_{2}\right)\right) \equiv l_{M}\left(\nu_{1}, \nu_{2}\right)$;

(ii) if $\nu \in H_{1}(M)$ and $\beta \in H_{2}(M)$, then $\alpha_{1}(\nu) \cdot \alpha_{2}(\beta)=\nu \cdot \beta$. A(M) will denote the group of all automorphisms.

Clearly any homeomorphism $f \in \mathscr{H}_{+}(M)$ induces an element $f_{*} \in A(M)$ and we let $H_{+}(M)$ be the subgroup of $A(M)$ consisting of all such automorphisms of $H_{*}(M)$.

By an algebraic morphism between two presentations $\left(\mathbf{Z}^{n}, L_{i}\right)(i=1,2)$ of $H_{*}(M)$, we shall mean a commutative diagram

$$
\begin{array}{rlrlrl}
0 \rightarrow & H_{2}(M) \stackrel{h_{1}}{\rightarrow} \mathbf{Z}^{n} \stackrel{\operatorname{ad}\left(L_{1}\right)}{\longrightarrow}\left[\mathbf{Z}^{n}\right]^{*} \stackrel{\partial_{1}}{\rightarrow} H_{1}(M) \rightarrow 0 \\
& \alpha_{2} \downarrow & \Lambda \downarrow & \uparrow \Lambda^{*} & & \downarrow \alpha_{1} \\
0 \rightarrow & H_{2}(M) \stackrel{h_{2}}{\rightarrow} \mathbf{Z}^{n} \stackrel{\operatorname{ad}\left(L_{2}\right)}{\longrightarrow}\left[\mathbf{Z}^{n}\right]^{*} \stackrel{\partial_{2}}{\rightarrow} H_{1}(M) \rightarrow 0
\end{array}
$$

where $\Lambda$ is an isometry. If both $\left(\mathbf{Z}^{n}, L_{1}\right)$ and $\left(\mathbf{Z}^{n}, L_{2}\right)$ are geometric and $\left(\alpha_{1}, \alpha_{2}\right) \in$ $H_{+}(M)$ we shall call the algebraic morphism simply a morphism. Such a diagram will be called a geometric morphism if $\Lambda$ is induced by a homeomorphism of the two manifolds.

Note that the isometry $\Lambda$ determines completely the algebraic morphism and so in particular we shall write $\left(\alpha_{1}, \alpha_{2}\right)=\partial(\Lambda)$. As $\Lambda$ preserves the pairings one may check that $\partial(\Lambda) \in A(M)$.

(1.3) Definition. Given a presentation $\left(\mathbf{Z}^{n}, L\right)$ of $H_{*}(M)$, let $A_{L}(M)$ be the subgroup of $A(M)$ given by

$$
A_{L}(M)=\left\{\partial(\Lambda) \mid \Lambda \text { a (self) isometry of }\left(\mathbf{Z}^{n}, L\right)\right\} .
$$

In general, $A_{L}(M)$ is not a normal subgroup of $A(M)$.

Define

$$
B_{L}(M)=H_{+}(M) \backslash A(M) / A_{L}(M) .
$$

The goal of the rest of this section is to better understand the quotients $A(M) / A_{L}(M)$ and $B_{L}(M)$. It turns out that they are determined by torsion information associated to $\left(\mathbf{Z}^{n}, L\right)$ in a way which we describe now.

Given a presentation $\left(\mathbf{Z}^{n}, L\right)$ of $H_{*}(M)$, there is an induced presentation of $\left(T_{1}(M), l_{M}\right)$ (in the sense of $\S 7$ of $[\mathbf{W 2}]$ ) as follows.

Set $K(L)=\operatorname{ker}(\operatorname{ad}(L))$ and let $\pi: \mathbf{Z}^{n} \rightarrow \mathbf{Z}^{n} / K(L)$ be the projection. $L$ induces a nonsingular pairing $L^{t}$ on $\mathbf{Z}^{n} / K(L)$ by the formula

$$
L^{t}\left(\pi\left(\xi_{1}\right), \pi\left(\xi_{2}\right)\right)=L\left(\xi_{1}, \xi_{2}\right) .
$$

It can be shown that there is a commutative diagram

$$
\begin{aligned}
& 0 \rightarrow \mathbf{Z}^{n} / K(L) \stackrel{\operatorname{ad}\left(L^{t}\right)}{\longrightarrow}\left[\mathbf{Z}^{n} / K(L)\right]^{*} \stackrel{\partial^{t}}{\rightarrow} T_{1}(M) \rightarrow 0 \\
& \pi \uparrow \quad \downarrow \pi^{*} \quad \downarrow i \\
& \mathbf{Z}^{n} \quad \stackrel{\operatorname{ad}(L)}{\longrightarrow} \quad\left[\mathbf{Z}^{n}\right]^{*} \quad \stackrel{\partial}{\rightarrow} H_{1}(M) \rightarrow 0
\end{aligned}
$$

where both rows are exact. The link pairing $l_{M}$ may be calculated from the top row by a formula similar to that in Definition (1.1)(i). 
(1.4) DEFINITION. Let $A^{t}(M)$ be the set of $l_{M}$-preserving isomorphism of $T_{1}(M), A_{L}^{t}$ the subgroup consisting of those elements induced by isometries of $\left(\mathbf{Z}^{n} / K(L), L^{t}\right)$ and $H_{+}^{t}(M)=\left\{f_{*}^{t} \mid f \in \nvdash_{+}(M)\right\} \subseteq A^{t}(M)$.

There is a restriction homomorphism $t: A(M) \rightarrow A^{t}(M)$ given by $t(\alpha)=\alpha_{1}^{t}$. It may be verified that $t\left(A_{1}(M)\right) \subseteq A_{L}^{t}(M)$ and clearly $t\left(H_{+}(M)\right)=H_{+}^{t}(M)$. Thus $t$ induces functions

(i) $t_{1}: A(M) / A_{L}(M) \rightarrow A^{t}(M) / A_{L}^{t}(M)$;

(ii) $t_{2}: B_{L}(M) \rightarrow B_{L}^{t}(M)=H_{+}^{t}(M) \backslash A^{t}(M) / A_{L}^{t}(M)$.

We shall show that both $t_{1}$ and $t_{2}$ are bijective.

(1.5) LEMMA. The functions $t, t_{1}$ and $t_{2}$ are epimorphisms.

ProOF. It suffices to show $t$ is surjective. To that end let $\alpha_{1}^{t} \in A^{t}(M)$ and extend $\alpha_{1}^{t}$ arbitrarily to an isomorphism $\alpha_{1}: H_{1}(M) \rightarrow H_{1}(M)$. There is an induced isomorphism $H_{1}(M) / T_{1}(M) \rightarrow H_{1}(M) / T_{1}(M)$ and we take $\alpha_{2}$ to be the inverse of its adjoint under the identification of $H_{1}(M)$ with $\left[H_{1}(M) / T_{1}(M)\right]^{*}$ resulting from Poincaré duality. It is not hard to check that $\left(\alpha_{1}, \alpha_{2}\right) \in A(M)$ and $t\left(\alpha_{1}, \alpha_{2}\right)=$ $\alpha_{1}^{t}$.

(1.6) Proposition. Let $\alpha \in A(M)$. Then $\alpha \in A_{L}(M) \Leftrightarrow \alpha_{1}^{t} \in A_{L}^{t}(M)$.

The proof of Proposition (1.6) will take up the rest of this section. Before proceeding to it we list various consequences.

(1.7) Corollary. The function $t_{1}: A(M) / A_{L}(M) \rightarrow A^{t}(M) / A_{L}^{t}(M)$ is bijective.

ProOF. Simply combine Lemmas (1.5) and (1.6).

(1.8) COROLlaRY. There is an integer $m>0$ depending only on $T_{1}(M)$ such that $\alpha^{m} \in A_{L}(M)$ for each $\alpha \in A(M)$.

PROOF. Let $m$ be an exponent for the group of isomorphisms of $T_{1}(M)$. Then for any $\alpha \in A(M), t\left(\alpha^{m}\right)=\left(\alpha_{1}^{t}\right)^{m}=1 \in A_{L}^{t}(M)$. Thus $\alpha^{m} \in A_{L}(M)$ by Proposition (1.6).

(1.9) COROLlaRY. Suppose $T_{1}(M) \cong \mathbf{Z} / r$ where $r=1,2,4, p^{n}$ or $2 p^{n}$, $p$ an odd prime. Then $A_{L}(M)=A(M)$.

Proof. When $T_{1}(M)$ is cyclic of order $r, A^{t}(M)=\left\{u \mid u^{2} \equiv 1\right\} \subseteq \mathbf{Z} / r$. But for the values of $r$ listed in the hypotheses, $u^{2} \equiv 1(\bmod r)$ implies $u \equiv \pm 1(\bmod r)$. Thus $A^{t}(M)=\{ \pm 1\}$. Now multiplication by \pm 1 is an isometry of $\left(\mathbf{Z}^{n} / K(L), L^{t}\right)$, from which we see that $A_{L}^{t}(M)=A^{t}(M)$. Corollary (1.7) now shows $A_{L}(M)=$ $A(M)$.

(1.10) COROLlary. The function $t_{2}: B_{L}(M) \rightarrow B_{L}^{t}(M)$ is bijective.

PROOF. As $t_{2}$ is surjective (Lemma (1.5)), we need only prove it is 1-1.

Let $\alpha, \beta \in A(M)$ be such that $t_{2}(\langle\alpha\rangle)=t_{2}(\langle\beta\rangle)$ (here \langle\rangle denotes equivalence class in $\left.B_{L}(M)\right)$. Then there is a homeomorphism $f \in \mathscr{H}_{+}(M)$ such that

$$
\left(\alpha^{-1} \circ f_{*}^{-1} \circ \beta\right)_{1}^{t}=\left(\alpha_{1}^{t}\right)^{-1} \circ\left(f_{*}^{t}\right)^{-1} \circ \beta_{1}^{t} \in A_{L}^{t}(M) \text {. }
$$

By Proposition (1.6), $\alpha^{-1} \circ f_{*}^{-1} \circ \beta \in A_{L}(M)$. Thus $\langle\alpha\rangle=\langle\beta\rangle$ and so $t_{2}$ is injective. We are therefore done. 
Now to the proof of Proposition (1.6). We need only show that $\alpha_{1}^{t} \in A_{L}^{t}(M)$ implies $\alpha \in A_{L}(M)$. Assume then that we are given a commutative diagram

$$
\begin{aligned}
& 0 \rightarrow \mathbf{Z}^{n} / K(L) \rightarrow\left[\mathbf{Z}^{n} / K(L)\right]^{*} \stackrel{\partial^{t}}{\rightarrow} T_{1}(M) \rightarrow 0 \\
& \Lambda^{t} \downarrow \quad \uparrow \Lambda^{t^{*}} \quad \downarrow \alpha_{1}^{t} \\
& 0 \rightarrow \mathbf{Z}^{n} / K(L) \rightarrow\left[\mathbf{Z}^{n} / K(L)\right]^{*} \stackrel{\partial^{t}}{\rightarrow} T_{1}(M) \rightarrow 0
\end{aligned}
$$

where $\Lambda^{t}$ is an isometry of $L^{t}$.

Let $\phi: \mathbf{Z}^{n} / K(L) \rightarrow \mathbf{Z}^{n}$ be an arbitrary splitting of $\pi$ and note that we may define an isometry $\Lambda$ of $\left(\mathbf{Z}^{n}, L\right)$ as in the diagram:

$$
\begin{array}{cc}
H_{2}(M) \oplus \mathbf{Z}^{n} / K(L) \stackrel{h+\phi}{\longrightarrow} \mathbf{Z}^{n} \\
\alpha_{2} \oplus \Lambda^{t} \downarrow & \\
H_{2}(M) \oplus \mathbf{Z}^{n} / K(L) \stackrel{h+\phi}{\longrightarrow} \mathbf{Z}^{n}
\end{array}
$$

(1.11) LEMMA . $\partial(\Lambda)=\left(\alpha_{1}+\psi, \alpha_{2}\right)$ where $\psi:\left(H_{1}(M), T_{1}(M)\right) \rightarrow\left(T_{1}(M), 0\right)$.

PROOF. That $\partial(\Lambda)_{2}=\alpha_{2}$ follows readily from the definition of $\Lambda$.

To show $\psi: H_{1}(M) \rightarrow T_{1}(M)\left(\psi=\partial(\Lambda)_{1}-\alpha_{1}\right)$, let $\nu \in H_{1}(M)$ and $\beta \in H_{2}(M)$ be arbitrary. Now

$$
\begin{aligned}
\psi(\nu) \cdot \alpha_{2}(\beta) & =\partial(\Lambda)_{1}(\nu) \cdot \alpha_{2}(\beta)-\alpha_{1}(\nu) \cdot \alpha_{2}(\beta) \\
& =\partial(\Lambda)_{1}(\nu) \cdot \partial(\Lambda)_{2}(\beta)-\alpha_{1}(\nu) \cdot \alpha_{2}(\beta) \\
& =\nu \cdot \beta-\nu \cdot \beta \quad \text { since } \partial(\Lambda), \alpha \in A(M) \\
& =0 .
\end{aligned}
$$

But as $\beta \in H_{2}(M)$ was arbitrary, we are forced to conclude $\psi(\nu) \in T_{1}(M)$. Thus image $(\psi) \subseteq T_{1}(M)$ as claimed.

Finally we show $\psi$ vanishes on $T_{1}(M)$. Now the identity $\pi \circ \Lambda=\Lambda^{t} \circ \pi$ implies that $\Lambda^{*} \circ \pi^{*}=\pi^{*} \circ \Lambda^{t^{*}}$. Hence, if $i: T_{1}(M) \rightarrow H_{1}(M)$ is the inclusion,

$$
\begin{aligned}
\alpha_{1} \circ i \circ \partial^{t} & =i \circ \alpha_{1}^{t} \circ \partial^{t}=i \circ \partial^{t} \circ\left(\Lambda^{t^{*}}\right)^{-1}=\partial \circ \pi^{*} \circ\left(\Lambda^{t^{*}}\right)^{-1} \\
& =\partial \circ \Lambda^{*^{-1}} \circ \pi^{*}=\partial(\Lambda)_{1} \circ \partial \circ \pi^{*}=\partial(\Lambda)_{1} \circ i \circ \partial^{t} .
\end{aligned}
$$

As $\partial^{t}$ is surjective, we conclude $\alpha_{1} \circ i=\partial(\Lambda)_{1} \circ i$. Thus $\psi\left(T_{1}(M)\right)=\{0\}$.

This completes the proof of the lemma.

The next lemma will show us how to alter $\Lambda$ to construct an isometry $\Lambda^{\prime}$ of $\left(\mathbf{Z}^{n}, L\right)$ with $\partial\left(\Lambda^{\prime}\right)=\alpha$.

(1.12) LEMMA. Let $\gamma: \mathbf{Z}^{n} / K(L) \rightarrow H_{2}(M)$ be an arbitrary homomorphism and set $\Lambda^{\prime}=\Lambda+h \circ \gamma \circ \pi$. Then

(i) $\Lambda^{\prime}$ is an isometry of $\left(\mathbf{Z}^{n}, L\right)$;

(ii) $\partial\left(\Lambda^{\prime}\right)_{2}=\alpha_{2}$

(iii) $\partial\left(\Lambda^{\prime}\right)_{1}^{-1}=\partial(\Lambda)_{1}^{-1}+\partial \circ \pi^{*} \circ \gamma^{*} \circ \mu$, where $\mu: H_{1}(M) \rightarrow H_{1}(M) / T_{1}(M)$ is the projection.

PROOF. We prove only (iii), the other two conclusions being straightforward.

Poincaré duality allows us to identify $H_{1}(M) / T_{1}(M)$ with $H_{2}(M)^{*}$ and under this identification, $h^{*}=\mu \circ \partial$. Thus $\Lambda^{\prime *}=\Lambda^{*}+\pi^{*} \circ \gamma^{*} \circ \mu \circ \partial$. Then

$\partial\left(\Lambda^{\prime}\right)_{1}^{-1} \circ \partial=\partial \circ \Lambda^{\prime *}=\partial \circ \Lambda^{*}+\partial \circ \pi^{*} \circ \gamma^{*} \circ \mu \circ \partial=\left(\partial(\Lambda)_{1}^{-1}+\partial \circ \pi^{*} \circ \gamma^{*} \circ \mu\right) \circ \partial$. 
The identity (iii) follows by noting that $\partial$ is surjective.

To choose the appropriate $\gamma$, use Lemma (1.11) to see that $\alpha_{1}^{-1}-\partial(\Lambda)_{1}^{-1}$ : $\left(H_{1}(M), T_{1}(M)\right) \rightarrow\left(T_{1}(M), 0\right)$. Thus we may construct a commutative diagram

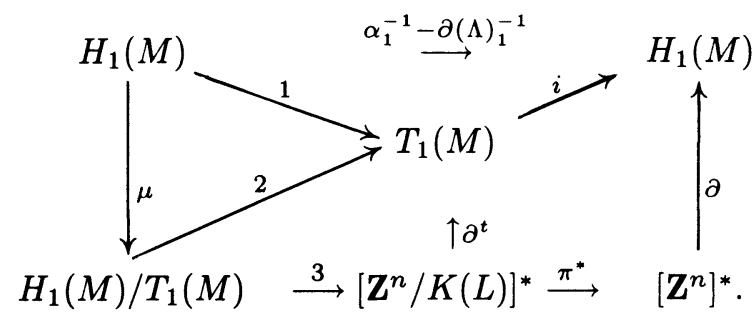

The numbers adjacent to several of the arrows refer to the order in which the corresponding homomorphisms are constructed.

Let $\gamma: \mathbf{Z}^{n} / K(L) \rightarrow H_{2}(M)$ be the adjoint of 3 . If $\Lambda^{\prime}$ is the associated isometry of $\left(\mathbf{Z}^{n}, L\right)$, as in Lemma (1.12), then

$$
\begin{aligned}
\partial\left(\Lambda^{\prime}\right)_{1}^{-1} & =\partial(\Lambda)_{1}^{-1}+\partial \circ \pi^{*} \circ \gamma^{*} \circ \mu \text { by Lemma (1.12)(iii) } \\
& =\partial(\Lambda)_{1}^{-1}+\left(\alpha_{1}^{-1}-\partial(\Lambda)_{1}^{-1}\right)=\alpha_{1}^{-1} .
\end{aligned}
$$

Hence $\partial\left(\Lambda^{\prime}\right)_{1}=\alpha_{1}$ and so $\alpha=\partial\left(\Lambda^{\prime}\right) \in A_{L}(M)$ as claimed. The proof of Proposition (1.6) is complete.

(1.13) REMARK. A more complete analysis of the quotient $A(M) / A_{L}(M)$ would involve $p$-local information. That is, the pairing $\left(\mathbf{Z}^{n} / K(L), L^{t}\right)$ would be tensored with $\mathbf{Z}_{p}$, the $p$-adic integers, and then the $p$-local analogues of $A^{t}(M), A_{L}^{t}(M)$ and their quotient determined. Presumably there is some sort of local to global correspondence which provides a complete set of invariants for $A(M) / A_{L}(M)$.

2. In this section we review some material on spin structures and the quadratic enhancements of the link pairing, $l_{M}$.

Let $\xi$ be a principal STop $(n)$-bundle $(n \geq 3)$ over a connected complex $X$, $\xi: \operatorname{STop}(n) \stackrel{i}{\rightarrow} E(\xi) \stackrel{\pi}{\rightarrow} X$.

(2.1) Definition. A spin structure on $\xi$ is a class $\sigma \in H^{1}(E(\xi) ; \mathbf{Z} / 2)$ which restricts to a generator of $H^{1}(\operatorname{STop}(n) ; \mathbf{Z} / 2) \cong \mathbf{Z} / 2$. The set of all spin structures on $\xi$ will be denoted $\operatorname{Spin}(\xi)$.

It follows from the Serre spectral sequence that $\xi$ admits a spin structure if and only if the sequence

$$
0 \rightarrow H^{1}(X ; \mathbf{Z} / 2) \stackrel{\pi^{*}}{\rightarrow} H^{1}(E(\xi) ; \mathbf{Z} / 2) \stackrel{i^{*}}{\rightarrow} H^{1}(\operatorname{STop}(n) ; \mathbf{Z} / 2) \rightarrow 0
$$

is exact. Notice that a spin structure on $\xi$ is just a splitting of this sequence from the right-hand side, that is a splitting of $i^{*}$.

Let $\sigma \in \operatorname{Spin}(\xi)$ and $x \in H^{1}(X ; \mathbf{Z} / 2)$. The formula

$$
x \cdot \sigma=\sigma+\pi^{*}(x)
$$

defines an effective, transitive action of $H^{1}(X ; \mathbf{Z} / 2)$ on $\operatorname{Spin}(\xi)$. For $\sigma_{1}, \sigma_{2} \in$ $\operatorname{Spin}(\xi)$, let

$$
d\left(\sigma_{1}, \sigma_{2}\right)=x \in H^{1}(X ; Z / 2) \text { if } x \cdot \sigma_{1}=\sigma_{2} .
$$


(2.2) REMARK. There is an analogous theory of spin structures on principal $\mathrm{SO}(n)$-bundles [Mi]. If $\xi$ is such a bundle, with $\xi_{0}$ the associated principal STop $(n)$ bundle, the inclusion $E(\xi) \rightarrow E\left(\xi_{0}\right)$ induces an equivalence of the $H^{1}(X ; \mathbf{Z} / 2)$-sets $\operatorname{Spin}(\xi)$ and $\operatorname{Spin}\left(\xi_{0}\right)$. Thus these two notions of spin structures on $\xi$ coincide and may be identified.

Now suppose that $X$ is a manifold with $\tau_{x}$ the principal STop $(n)$-bundle associated to the stable topological tangent bundle of $X$.

(2.3) DEFINITION. By a spin structure on $X$, we shall mean a spin structure on $\tau_{x}$. The set $\operatorname{Spin}\left(\tau_{x}\right)$ will be denoted simply by $\operatorname{Spin}(X)$.

There is a left-action of $\mathscr{H}_{+}(X)$ on $\operatorname{Spin}(X)$. Each $f \in \mathscr{H}_{+}(X)$ determines a bundle map (up to bundle isotopy)

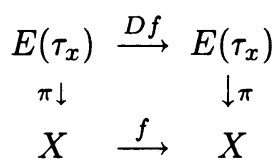

and so we may define

$$
f_{\#}=\left(D f^{*}\right)^{-1}: \operatorname{Spin}(X) \rightarrow \operatorname{Spin}(X) .
$$

(2.4) Proposition. For any $f \in \nvdash_{+}(X), \sigma \in \operatorname{Spin}(X)$ and $x \in H^{1}(X ; Z / 2)$, $x \cdot f_{\#}(\sigma)=f_{\#}\left(f^{*}(x) \cdot \sigma\right)$.

It is worth mentioning that isotopic homeomorphisms induce the same permutation of $\operatorname{Spin}(X)$. This observation allows one to calculate the action of $f \in \mathcal{H}_{+}(M)$ on $\operatorname{Spin}(M)$ from the action of some diffeomorphism isotopic to $f$ on the stable $\mathrm{SO}(n)$ spin structures of $M$.

Through the use of Wu's formula (see [Wu] for the absolute case and $\S 7$ of $[\mathbf{K}]$ for the relative version) it can be shown that a (1-connected) 4-manifold $V$ admits a spin structure if and only if $\left(H_{2}(V), \cdot\right)$ is an even pairing. If there is a spin structure on $V$, it is necessarily unique.

(2.5) DEFINITION. Let $V$ be a 1-connected 4-manifold with boundary $M$ and even intersection pairing. The spin structure $\sigma_{V} \in \operatorname{Spin}(M)$ is defined to be the restriction to $M$ of the unique spin structure on $V$.

Turning things around, we can ask what the obstruction to extending a given spin structure $\sigma \in \operatorname{Spin}(M)$ across a 1-connected 4-manifold $V$ with boundary $M$ is. There is a commutative diagram

$$
\begin{array}{ccc}
H^{1}\left(E\left(\tau_{M}\right) ; \mathbf{Z} / 2\right) & \stackrel{\delta_{E}}{\longrightarrow} H^{2}\left(E\left(\tau_{V}\right), E\left(\tau_{M}\right) ; \mathbf{Z} / 2\right) \\
\pi^{*} \uparrow & & \cong \uparrow \pi^{*} \\
H^{1}(M ; \mathbf{Z} / 2) & \stackrel{\delta}{\longrightarrow} & H^{2}(V, M ; \mathbf{Z} / 2)
\end{array}
$$

(2.6) Definition. The relative Stiefel-Whitney class $w_{2}(V, M ; \sigma)$, is the element $\left(\pi^{*}\right)^{-1} \circ \delta_{E}(\sigma) \in H^{2}(V, M ; \mathbf{Z} / 2)$.

Our terminology is justified because, through the identification of $\operatorname{Spin}(M)$ with vertical homotopy classes of sections of $\tau_{M^{*}}, M^{*}=M \backslash\{*\}$, (see alternate Definition 2 in $[\mathbf{M i}]), w_{2}(V, M ; \sigma)$ is precisely the second relative Stiefel-Whitney class associated to $\sigma$ (see $[\mathbf{K}]$ ). 
It can be shown that if $j:(V, \phi) \rightarrow(V, M)$ is the inclusion, then $j^{*} w_{2}(V, M ; \sigma)=$ $w_{2}(V)$ for each $\sigma \in \operatorname{Spin}(M)$.

(2.7) Lemma. The function $w_{2}(V, M): \operatorname{Spin}(M) \rightarrow H^{2}(V, M ; \mathbf{Z} / 2)$ satisfies the following:

(i) image $\left(w_{2}(V, M)\right)=\left(j^{*}\right)^{-1}\left(w_{2}(V)\right)$;

(ii) a class $w \in H^{2}(V, M ; \mathbf{Z} / 2)$ lies in image $\left(w_{2}(V, M)\right)$ if and only if $\left\langle w, j_{*}(\xi)\right\rangle \equiv$ $\xi \cdot \xi(\bmod 2)$ for each $\xi \in H_{2}(V)$;

(iii) if $x \in H^{1}(M ; \mathbf{Z} / 2)$ and $\sigma \in \operatorname{Spin}(M)$, then

$$
w_{2}(V, M ; x \cdot \sigma)=w_{2}(V, M ; \sigma)+\delta x .
$$

Thus $w_{2}(V, M)$ is injective;

(iv) $w_{2}(V, M ; \sigma)=0$ if and only if $\left(H_{2}(V), \cdot\right)$ is even and $\sigma=\sigma_{V}$.

We omit the proof of this lemma as most of it follows in a straightforward fashion from what has been done previously. We do mention though that to prove (ii) one needs (i) together with Wu's formula.

It will be convenient for us to deal with the function $\gamma$ : $\operatorname{Spin}(M) \rightarrow H_{2}(V ; \mathbf{Z} / 2)$ dual to $w_{2}(V, M)$. That is,

$$
\gamma(\sigma)=D_{V} w_{2}(V, M ; \sigma)
$$

$D_{V}: H^{2}(V, M ; \mathbf{Z} / 2) \rightarrow H_{2}(V ; \mathbf{Z} / 2)$ being the Lefschetz duality isomorphism.

The function $\gamma$ has a more elegant formulation as the composition

$$
\operatorname{Spin}(M) \rightarrow \Omega_{3}^{\text {spintop }}(V) \cong H_{2}(V) \otimes \Omega_{1}^{\text {spintop }}=H_{2}(V ; \mathbf{Z} / 2),
$$

where the function $\operatorname{Spin}(M) \rightarrow \Omega_{3}^{\text {spintop }}(V)$ associates the singular spin manifold " $M^{\sigma} \rightarrow V$ " to $\sigma \in \operatorname{Spin}(M)$.

Let $D_{M}: H^{1}(M ; \mathbf{Z} / 2) \rightarrow H_{2}(M ; \mathbf{Z} / 2)$ be the Poincaré isomorphism and $h: M \rightarrow$ $V$ the inclusion. The next lemma is an easy consequence of Lemma (2.7).

(2.8) LEMMA. The function $\gamma: \operatorname{Spin}(M) \rightarrow H_{2}(V ; \mathbf{Z} / 2)$ satisfies:

(i) a class $\xi \in H_{2}(V ; \mathbf{Z} / 2)$ lies in image $(\gamma)$ if and only if $\xi \cdot \xi_{1} \equiv \xi_{1} \cdot \xi_{1}(\bmod 2)$ for each $\xi_{1} \in H_{2}(V)$;

(ii) for $x \in H^{1}(M ; \mathbf{Z} / 2)$ and $\sigma \in \operatorname{Spin}(M)$,

$$
\gamma(x \cdot \sigma)=\gamma(\sigma)+h_{*} D_{M}(x)
$$

In particular, $\gamma$ is injective.

(2.9) LemMA. Let $V_{1}$ and $V_{2}$ be two 1-connected 4-manifolds with boundary $M$ and $\Lambda$ an isometry of their intersection pairings. Then there is a permutation $\pi_{\Lambda}$ of $\operatorname{Spin}(M)$ such that

(i) $\gamma_{2}\left(\pi_{\Lambda}(\sigma)\right)=\Lambda\left(\gamma_{1}(\sigma)\right), \sigma \in \operatorname{Spin}(M)$;

(ii) $\pi_{\Lambda}\left(\partial(\Lambda)_{1}^{*}(x) \cdot \sigma\right)=x \cdot \pi_{\Lambda}(\sigma)$ for each $x \in H^{1}(M ; \mathbf{Z} / 2)$ and $\sigma \in \operatorname{Spin}(M)$.

Proof. As $\Lambda$ is an isometry, we can use Lemma (2.8)(i) to see that $\Lambda$ (image $\left(\gamma_{1}\right)$ ) $=\operatorname{image}\left(\gamma_{2}\right)$. As $\gamma_{2}$ is injective (Lemma (2.8)(ii)), we may define $\pi_{\Lambda}=\gamma_{2}^{-1} \circ \Lambda \circ \gamma_{1}$. Evidently (i) holds.

To prove (ii), we first note that the composition $D_{M} \circ \partial(\Lambda)_{1}^{*} \circ D_{M}^{-1}$ defines an isomorphism of $H_{2}(M ; \mathbf{Z} / 2)$ which we denote (through abuse of notation) as 
$\partial(\Lambda)_{2}^{-1}$. It can be checked that $\Lambda \circ h_{1 *}=h_{2 *} \circ \partial(\Lambda)_{2}$. Then for any $x \in H^{1}(M ; \mathbf{Z} / 2)$ and $\sigma \in \operatorname{Spin}(M)$

$$
\begin{aligned}
\gamma_{2}\left(\pi_{\Lambda}\left(\partial(\Lambda)_{1}^{*}(x) \cdot \sigma\right)\right) & =\Lambda\left(\gamma_{1}(\sigma)+h_{1 *} \circ D_{M}\left(\partial(\Lambda)_{1}^{*}(x)\right)\right) \\
& =\gamma_{2}\left(\pi_{\Lambda}(\sigma)\right)+h_{2 *} \circ \partial(\Lambda)_{2} \circ D_{M} \circ \partial(\Lambda)_{1}^{*}(x) \\
& =\gamma_{2}\left(\pi_{\Lambda}(\sigma)\right)+h_{2 *} \circ D_{M}(x)=\gamma_{2}\left(x \cdot \pi_{\Lambda}(\sigma)\right)
\end{aligned}
$$

As $\gamma_{2}$ is injective, identity (ii) follows.

We close this section with a short discussion of the quadratic functions related to $l_{M}$.

To each $\sigma \in \operatorname{Spin}(M)$, there is an associated quadratic enhancement of $l_{M}$ (see $\S 4$ of $[\mathbf{T a}])$, that is a function $q_{\sigma}: T_{1}(M) \rightarrow \mathbf{Q} / \mathbf{Z}$ satisfying

(i) $q_{\sigma}\left(z_{1}+z_{2}\right) \equiv q_{\sigma}\left(z_{1}\right)+q_{\sigma}\left(z_{2}\right)+l_{M}\left(z_{1}, z_{2}\right), z_{1}, z_{2} \in T_{1}(M)$;

(ii) $q_{\sigma}(m z) \equiv m^{2} q_{\sigma}(z), z \in T_{1}(M)$ and $m \in \mathbf{Z}$.

Moreover, any function $q: T_{1}(M) \rightarrow \mathbf{Q} / \mathbf{Z}$ satisfying these two equations is of the form $q=q_{\sigma}$ for some spin structure $\sigma$ (see Corollary (4.3) of [Ta]).

Now for any $x \in H^{1}(M ; \mathbf{Z} / 2), \sigma \in \operatorname{Spin}(M)$ and $z \in T_{1}(M)$,

$$
q_{x \cdot \sigma}(z)=q_{\sigma}(z)+\frac{1}{2}\langle x, z\rangle
$$

where $\frac{1}{2}\langle x, z\rangle$ is defined to be $\frac{1}{2}$ when $\langle x, z\rangle \equiv 1(\bmod 2)$ and zero otherwise. Thus, if $I^{1}(M)$ is the image of $H^{1}(M)$ in $H^{1}(M ; \mathbf{Z} / 2)$ under $(\bmod 2)$ reduction, we have

(2.10) LEMMA. $q_{\sigma_{1}}=q_{\sigma_{2}}$ if and only if $d\left(\sigma_{1}, \sigma_{2}\right) \in I^{1}(M)$.

(2.11) Proposition. An even bilinear form space $\left(\mathbf{Z}^{n}, L\right)$ presenting $H_{*}(M)$ determines a subset $\operatorname{Spin}_{L}(M)$ of $\operatorname{Spin}(M)$ of the form $\operatorname{Spin}_{L}(M)=I^{1}(M) \cdot \sigma$ for some spin structure $\sigma$ on $M$.

Proof. Let $\xi \in \mathbf{Z}^{n}$ and $m \eta=\operatorname{ad}(L)(\xi)$ for some $\eta \in\left[\mathbf{Z}^{n}\right]^{*}$ and $m \neq 0$. As $\left(\mathbf{Z}^{n}, L\right)$ presents $H_{*}(M)$, it can be verified that the formula

$$
q(\partial \eta) \equiv-L(\xi, \xi) / 2 m^{2}
$$

determines a quadratic enhancement of $l_{M}$. Set

$$
\operatorname{Spin}_{L}(M)=\left\{\sigma \mid q_{\sigma}=q\right\} \subseteq \operatorname{Spin}(M) .
$$

According to Lemma (2.10), $\operatorname{Spin}_{L}(M)$ is precisely an orbit of the $I^{1}(M)$-action on $\operatorname{Spin}(M)$. This completes the proof.

(2.12) REMARK. (i) Let $M=\partial V$ where $V$ is a 1-connected 4-manifold. If $\xi \in H_{2}(V)$ with $j_{*}(\xi)=m \eta, \eta \in H_{2}(V, M)$ and $m \neq 0$, then it can be argued using the geometric description of $q_{\sigma}$ that

$$
q_{\sigma}(\partial \eta) \equiv-\frac{1}{2 m^{2}}(\xi \cdot \xi)+\frac{1}{2}\left\langle w_{2}(V, M ; \sigma), \eta\right\rangle
$$

If $V$ is actually a spin manifold, then Lemma (2.7)(iv) shows that $\operatorname{Spin}_{L}(M)=$ $I^{1}(M) \cdot \sigma_{V}$.

(ii) The identity

$$
\left\langle w_{2}\left(V_{2}, M ; \pi_{\Lambda}(\sigma)\right), \eta\right\rangle \equiv\left\langle w_{2}\left(V_{1}, M ; \sigma\right), \Lambda^{*}(\eta)\right\rangle,
$$

$\eta \in H_{2}(V, M)$, together with (II.3) imply that for any $\sigma \in \operatorname{Spin}(M)$ and $z \in T_{1}(M)$,

$$
q_{\pi_{\Lambda}(\sigma)}\left(\partial(\Lambda)_{1}(z)\right) \equiv q_{\sigma}(z) .
$$

(Compare Lemma (3.1)(iv).) 
3. This section provides the more detailed structure necessary to the understanding of $\mathcal{V}_{L}(M)$ when $L$ is even. We shall assume then that all forms $\left(\mathbf{Z}^{n}, L\right)$ are even, present $H_{*}(M)$ and that we have fixed some $\sigma_{L} \in \operatorname{Spin}_{L}(M)$ such that $\sigma_{L}=\sigma_{V}$ whenever $\left(\mathbf{Z}^{n}, L\right)=\left(H_{2}(V), \cdot\right), V$ a 1-connected 4-manifold with boundary $M$.

Define $\gamma_{L}: \operatorname{Spin}(M) \rightarrow \mathbf{Z}^{n} \otimes \mathbf{Z} / 2$ by the formulua $\gamma_{L}\left(x \cdot \sigma_{L}\right)=h\left(D_{M}(x)\right)$. Here $h: H_{2}(M ; \mathbf{Z} / 2) \rightarrow \mathbf{Z}^{n} \otimes \mathbf{Z} / 2$ is the homomorphism adjoint to $(\partial \otimes 1):\left[\mathbf{Z}^{n}\right]^{*} \otimes \mathbf{Z} / 2 \rightarrow$ $H_{1}(M ; \mathbf{Z} / 2)$.

When $\left(\mathbf{Z}^{n}, L\right)$ is geometric, $\gamma_{L}$ coincides with the function $\gamma$ defined in $\S 2$. In general, it satisfies the appropriate analogue of Lemma (2.8).

(3.1) LEMMA. Let $\Lambda:\left(\mathbf{Z}^{n}, L_{1}\right) \rightarrow\left(\mathbf{Z}^{n}, L_{2}\right)$ be an isometry of even bilinear form spaces presenting $H_{*}(M)$. Then there is a permutation $\pi_{\Lambda}: \operatorname{Spin}(M) \rightarrow \operatorname{Spin}(M)$ such that

(i) $\gamma_{L_{2}}\left(\pi_{\Lambda}(\sigma)\right)=\Lambda\left(\gamma_{L_{1}}(\sigma)\right)$. In particular $\pi_{\Lambda}$ agrees with the permutation of Lemma (2.9) when $\left(\mathbf{Z}^{n}, L_{1}\right)$ and $\left(\mathbf{Z}^{n}, L_{2}\right)$ are geometric;

(ii) $\pi_{\Lambda}\left(\sigma_{L_{1}}\right)=\sigma_{L_{2}}$;

(iii) $\pi_{\Lambda}\left(\partial(\Lambda)_{1}^{*}(x) \cdot \sigma\right)=x \cdot \pi_{\Lambda}(\sigma)$, where $x \in H^{1}(M ; \mathbf{Z} / 2)$ and $\sigma \in \operatorname{Spin}(M)$;

(iv) $q_{\pi_{\Lambda}(\sigma)}\left(\partial(\Lambda)_{1}(z)\right)=q_{\sigma}(z)$ for $z \in T_{1}(M), \sigma \in \operatorname{Spin}(M)$.

ProOF. Proceed as in Lemma (2.9) to construct a $\pi_{\Lambda}$ satisfying (i) and (iii). It is easily checked that $\pi_{\Lambda}\left(\sigma_{L_{1}}\right)=\sigma_{L_{2}}$ so (ii) holds.

Finally for (iv), use the fact that $\Lambda$ is an isometry together with (ii) and the definitions of $q_{\sigma_{L_{1}}}$ and $q_{\sigma_{L_{2}}}$ (Proposition (2.11)) to see that the equation holds for $\sigma=\sigma_{L_{1}}$. The general case follows from this, part (iii) and equation (II.2).

We use the material above to enhance the group $A(M)$. Fix a presentation $\left(\mathbf{Z}^{n}, L\right)$ of $H_{*}(M)$ and let $S(\operatorname{Spin}(M))$ denote the symmetric group on $\operatorname{Spin}(M)$.

(3.2) Definition. The group $\hat{A}(M)$ is the set of all pairs $(\alpha, \pi) \in A(M) \times$ $S(\operatorname{Spin}(M))$ such that

(i) $\pi\left(\alpha_{1}^{*}(x) \cdot \sigma\right)=x \cdot \pi(\sigma)$ for each $x \in H^{1}(M ; \mathbf{Z} / 2), \sigma \in \operatorname{Spin}(M)$;

(ii) $q_{\pi(\sigma)}\left(\alpha_{1}(z)\right)=q_{\sigma}(z)$ for each $z \in T_{1}(M)$ and $\sigma \in \operatorname{Spin}(M)$.

For any $(1, \pi) \in \hat{A}(M)$, it follows from Lemma $(2.10)$ that $\pi(\sigma)=y \cdot \sigma$ for some $y \in I^{1}(M)$. Conversely, any such pair $(1, y) \in \hat{A}(M)$. Thus we have an embedding $I^{1}(M) \rightarrow \hat{A}(M)$ whose image is easily verified to be normal.

Let $p: \hat{A}(M) \rightarrow A(M)$ be the projection.

(3.3) LEMMA. There is an exact sequence

$$
1 \rightarrow I^{1}(M) \rightarrow \hat{A}(M) \stackrel{p}{\rightarrow} A(M) \rightarrow 1 .
$$

ProOF. The only thing left to prove is that $p$ is surjective. But for $\alpha \in A(M)$, the function $q_{\sigma_{L}} \cdot \alpha_{1}^{-1}$ is a quadratic enhancement of $l_{M}$. Thus there is some $\sigma \in \operatorname{Spin}(M)$ with $q_{\sigma}=q_{\sigma_{L}} \cdot \alpha_{1}^{-1}$. Define $\pi \in S(\operatorname{Spin}(M))$ by the formula

$$
\pi\left(\alpha_{1}^{*}(x) \cdot \sigma_{L}\right)=x \cdot \sigma, \quad x \in H^{1}(M ; \mathbf{Z} / 2) .
$$

It is readily checked that $(\alpha, \pi) \in \hat{A}(M)$. Thus $p$ is surjective. 
Define subgroups $\hat{A}_{L}(M)$ and $\hat{H}_{+}(M)$ of $\hat{A}(M)$ by setting

$$
\begin{aligned}
& \hat{A}_{L}(M)=\left\{\left(\partial(\Lambda), \pi_{\Lambda}\right) \mid \Lambda \text { an isometry of }\left(\mathbf{Z}^{n}, L\right)\right\} ; \\
& \hat{H}_{+}(M)=\left\{\left(f_{*}, f_{\#}\right) \mid f \in \mathscr{H}_{+}(M)\right\} .
\end{aligned}
$$

That $\hat{A}_{L}(M) \subseteq \hat{A}(M)$ is Lemma (3.1). That $\hat{H}_{+}(M) \subseteq \hat{A}(M)$ is a consequence of Proposition (2.4) and the geometric description of $q_{\sigma}$ (see $\S 4$ of $[\mathrm{Ta}]$ ).

Clearly $p\left(\hat{A}_{L}(M)\right)=A_{L}(M)$ and $p\left(\hat{H}_{+}(M)\right)=H_{+}(M)$. Thus if $\hat{B}_{L}(M)=$ $\hat{H}_{+}(M) \backslash \hat{A}(M) / \hat{A}_{L}(M)$, the projection $p: \hat{A}(M) \rightarrow A(M)$ induces a function $p_{1}$ : $\hat{B}_{L}(M) \rightarrow B_{L}(M)$. It is possible to identify precisely the fibers of $p_{1}$. To that end, let $\alpha \in A(M)$ and define a subgroup $\sharp_{+}(M, \alpha)$ of $\sharp_{+}(M)$ by

$$
\mathscr{H}_{+}(M, \alpha)=\left\{f \mid f_{*} \in \alpha A_{L}(M) \alpha^{-1}\right\} .
$$

The symbol "( )" will denote equivalence class in both $B_{L}(M)$ and $\hat{B}_{L}(M)$.

(3.4) Proposition. For any $(\alpha, \pi) \in \hat{A}(M)$, the action of $\mathcal{H}_{+}(M, \alpha)$ on $\operatorname{Spin}(M)$ preserves $\pi\left(\operatorname{Spin}_{L}(M)\right)$. Further, there is a bijection

$$
\psi_{(\alpha, \pi)}: \pi\left(\operatorname{Spin}_{L}(M)\right) / \not_{+}(M, \alpha) \rightarrow p_{1}^{-1}(\langle\alpha\rangle) .
$$

Before proving this proposition we derive a few of its consequences. The first we look at gives a bound on the size of the fibers of $p_{1}$.

Consider $f \in \mathscr{H}_{+}(M)$ with $f_{*}= \pm 1$. As $p_{1}\left(f_{*}, f_{\#}\right)=p_{1}( \pm 1,1)$, we may apply Lemma (3.3) to see that $f_{\#}=y$ for some $y \in I^{1}(M)$. Thus we may consider $J=\left\{f_{\#} \mid f_{*}= \pm 1\right\}$ as a subset of $I^{1}(M)$. It is not hard to verify that $J$ is actually a subgroup of $I^{1}(M)$.

(3.5) Corollary. For any $\alpha \in A(M),\left|p_{1}^{-1}(\langle\alpha\rangle)\right| \leq 2^{b_{1}(M)} /|J|$.

Proof. Choose $\pi \in S(\operatorname{Spin}(M))$ such that $(\alpha, \pi) \in \hat{A}(M)$. Now clearly $\left\{f \mid f_{*}=\right.$ $\pm 1\} \subseteq \not_{+}(M, \alpha)$ so that

$$
\left|p_{1}^{-1}(\langle\alpha\rangle)\right|=\left|\pi\left(\operatorname{Spin}_{L}(M)\right) / \mathscr{H}_{+}(M, \alpha)\right| \leq\left|\pi\left(\operatorname{Spin}_{L}(M)\right) / J\right|=2^{b_{1}(M)} /|J| .
$$
tion.

(3.6) CoRollary. If $H_{1}(M ; \mathbf{Q}) \cong 0$, then $p_{1}: \hat{B}_{L}(M) \rightarrow B_{L}(M)$ is a bijec-

(3.7) Corollary. If $T_{1}(M)=0$, then $\hat{B}_{L}(M) \cong \operatorname{Spin}(M) / H_{+}(M)$.

Proof. If $T_{1}(M)=0$ then

(i) $\operatorname{Spin}_{L}(M)=\operatorname{Spin}(M)$;

(ii) $A_{L}(M)=A(M)$ (by Corollary (1.7)).

Thus $\left|B_{L}(M)\right|=1$ and $\mathscr{H}_{+}(M, \alpha)=\mathscr{H}_{+}(M)$ for each $\alpha \in A(M)$. In particular,

$$
\hat{B}_{L}(M)=p_{1}^{-1}(\langle 1\rangle) \cong \operatorname{Spin}(M) / \mathscr{H}_{+}(M) \text {. }
$$

Proof of Proposition (3.4). First we show $\mathfrak{H}_{+}(M, \alpha)$ acts on $\pi\left(\operatorname{Spin}_{L}(M)\right)$.

For $f \in \mathscr{H}_{+}(M, \alpha)$, there is an isometry $\Lambda$ of $\left(\mathbf{Z}^{n}, L\right)$ such that $f_{*}=\alpha \partial(\Lambda) \alpha^{-1}$. According to Lemma (3.3), there is a $y \in I^{1}(M)$ such that $f_{\#}=y \cdot \pi \circ \pi_{\Lambda} \circ \pi^{-1}$. As $\pi_{\Lambda}\left(\operatorname{Spin}_{L}(M)\right)=\operatorname{Spin}_{L}(M)(\operatorname{Lemma}(3.1))$, it is evident that $f_{\#}$ preserves $\pi\left(\operatorname{Spin}_{L}(M)\right)$.

Tentatively define

$$
\psi_{(\alpha, \pi)}: \pi\left(\operatorname{Spin}_{L}(M)\right) / \nvdash_{+}(M, \alpha) \rightarrow p_{1}^{-1}(\langle\alpha\rangle)
$$


by the formula

$$
\psi_{(\alpha, \pi)}\left(\overline{y \cdot \pi\left(\sigma_{L}\right)}\right)=\langle\alpha, y \cdot \pi\rangle
$$

where the bar denotes the equivalence class of $y \cdot \pi\left(\sigma_{L}\right)$.

To see this is well defined, let $f \in \nvdash_{+}(M, \alpha)$ and suppose $y_{2} \cdot \pi\left(\sigma_{L}\right)=$ $f_{\#}\left(y_{1} \cdot \pi\left(\sigma_{L}\right)\right)$. Choose an isometry $\Lambda$ such that $f_{*}=\alpha \circ \partial\left(\Lambda^{-1}\right) \circ \alpha^{-1}$. Then $\alpha=f_{*} \circ \alpha \circ \partial(\Lambda)$ and so there is some $y \in I^{1}(M)$ with

$$
y_{2} \cdot \pi=y \cdot f_{\#} \circ\left(y_{1} \cdot \pi\right) \circ \pi_{\Lambda} .
$$

From Lemma (3.1)(ii), $\pi_{\Lambda}\left(\sigma_{L}\right)=\sigma_{L}$, hence evaluating (III.1) at $\sigma_{L}$ shows $y=0$. Then $\left\langle\alpha, y_{1} \cdot \pi\right\rangle=\left\langle\alpha, y_{2} \cdot \pi\right\rangle$ which implies $\psi_{(\alpha, \pi)}$ is well defined.

That $\psi_{(\alpha, \pi)}$ is onto is a straightforward consequence of Lemma (3.3).

Finally, $\psi_{(\alpha, \pi)}$ is $1-1$ essentially by the definition of $\hat{B}_{L}(M)$.

The proof is now complete.

We close this section by remarking that as in the analysis of $B_{L}(M), \hat{B}_{L}(M)$ may be calculated from certain torsion information. Indeed let

$$
\begin{aligned}
\hat{A}^{t}(M) & =\left\{\left(\alpha_{1}^{t}, \pi\right) \mid \text { there is some }(\alpha, \pi) \in \hat{A}(M) \text { restricting to }\left(\alpha_{1}^{t}, \pi\right)\right\} ; \\
A_{L}^{t}(M) & =\left\{\left(\partial(\Lambda)_{1}^{t}, \pi_{\Lambda}\right) \mid \Lambda \text { is an isometry of }\left(\mathbf{Z}^{n}, L\right)\right\} ; \\
\hat{H}_{+}^{t} & =\left\{\left(f_{*}^{t}, f_{\#}\right) \mid f \in H_{+}(M)\right\} ; \\
\hat{B}_{L}^{t}(M) & =\hat{H}_{+}^{t}(M) \backslash \hat{A}^{t}(M) / \hat{A}_{L}^{t}(M) .
\end{aligned}
$$

As in $\S 1$, it can be shown that $\hat{A}(M) / \hat{A}_{L}(M) \cong \hat{A}^{t}(M) / \hat{A}_{L}^{t}(M)$ and $\hat{B}_{L}(M) \cong$ $\hat{B}_{L}^{t}(M)$.

4. Throughout this section $V_{1}$ and $V_{2}$ will be two 1-connected 4-manifolds with boundary $M$ and $(f, \Lambda): V_{1} \rightarrow V_{2}$ a fixed morphism. As $f_{*}=\partial(\Lambda),(2.9)(\mathrm{ii}),(2.10)$ and (2.12)(ii) show there is a class $\theta(f, \Lambda) \in I^{1}(M)$ such that $f_{\#}=\theta(f, \Lambda) \cdot \pi_{\Lambda}$. (Compare Lemma (3.3).)

(4.1) Proposition. The class $\theta(f, \Lambda)$ satisfies the following conditions.

(i) Let $\sigma \in \operatorname{Spin}(M)$ and $\delta_{2}: H^{1}(M ; \mathbf{Z} / 2) \rightarrow H^{2}\left(V_{2}, M ; \mathbf{Z} / 2\right)$ be the coboundary. Then

$$
\delta_{2} \theta(f, \Lambda)=w_{2}\left(V_{2}, M ; f_{\#}(\sigma)\right)-w_{2}\left(V_{2}, M ; \pi_{\Lambda}(\sigma)\right) .
$$

(ii) If $(g, \Psi): V_{2} \rightarrow V_{3}$ is another morphism, then

$$
\theta(g \circ f, \Psi \circ \Lambda)=\theta(g, \Psi)+\left(g^{-1}\right)^{*} \theta(f, \Lambda) .
$$

(iii) $\theta\left(f^{-1}, \Lambda^{-1}\right)=f^{*} \theta(f, \Lambda)$.

(iv) If $V_{1}=V_{2}$, there is an $m>0$ depending only on $b_{1}(M)=\operatorname{dim}_{\mathbf{Z} / 2}\left(I^{1}(M)\right)$ such that $\theta\left(f^{m}, \Lambda^{m}\right)=0$.

(v) If $\left(H_{2}\left(V_{i}\right), \cdot\right)$ is even $(i=1,2)$, then

$$
\theta(f, \Lambda)=d\left(f_{\#}\left(\sigma_{V_{1}}\right), \sigma_{V_{2}}\right) .
$$

Hence $\theta(f, \Lambda)$ depends only on $f$.

(vi) If $\left(H_{2}\left(V_{i}\right), \cdot\right)$ is even $(i=1,2)$ and $\theta(f, \Lambda)=0$, then $\Delta\left(V_{1}\right) \equiv \Delta\left(V_{2}\right)$.

Proof. For any $\sigma \in \operatorname{Spin}(M), \theta(f, \Lambda)=d\left(f_{\#}(\sigma), \pi_{\Lambda}(\sigma)\right)$. The identity (i) now follows from Lemma (2.7)(iii).

Conclusions (ii) and (iii) are ready consequences of Proposition (2.4). 
To deduce (iv), choose $m=2 m_{1}, m_{1}=\left|\operatorname{Gl}\left(b_{1}(M), \mathbf{Z} / 2\right)\right|$. Then $\left[f^{*} \mid I^{1}(M)\right]^{m_{1}}=$ 1 and therefore by (ii),

$$
\theta\left(f^{m}, \Lambda^{m}\right)=\theta\left(f^{m_{1}}, \Lambda^{m_{1}}\right)+\left(f^{*}\right)^{-m_{1}} \theta\left(f^{m_{1}}, \Lambda^{m_{1}}\right)=0 .
$$

For the identity in (v), use Lemma (3.1)(ii) to see $\pi_{\Lambda}\left(\sigma_{V_{1}}\right)=\sigma_{V_{2}}$. The result is then obvious.

Finally we prove (vi). Now according to (v), $\theta(f, \Lambda)=0$ implies $f_{\#}\left(\sigma_{V_{1}}\right)=\sigma_{V_{2}}$. Then for any smoothable spin, 4-manifold $V$ with boundary $M$ and $\sigma_{V}=\sigma_{V_{2}}$, consideration of the mapping cylinder of the composition $M \stackrel{f}{\rightarrow} M \rightarrow V$ shows that

$$
\mu\left(M ; \sigma_{V_{1}}\right) \equiv \mu\left(M ; \sigma_{V_{2}}\right) \quad(\bmod 16) .
$$

Here $\mu(M): \operatorname{Spin}(M) \rightarrow \mathbf{Z} / 16$ is the Rohlin function [Ka]. But using Theorem (13.1) of $[\mathbf{S}]$, it can be shown that

$$
\Delta\left(V_{i}\right) \equiv \frac{1}{8}\left[\text { signature } V_{i}-\mu\left(M ; \sigma_{V_{i}}\right)\right] \quad(\bmod 2) .
$$

Thus $\Delta\left(V_{1}\right) \equiv \Delta\left(V_{2}\right)$ as claimed.

This completes the proof of Proposition (4.1).

Our goal in this section is to prove that $\theta(f, \Lambda)$ is precisely the obstruction to realizing $(f, \Lambda)$ geometrically by a homeomorphism $F: V_{1} \rightarrow V_{2}$. That there is an obstruction is readily seen by taking $V_{1}=V_{2}=B^{2} \times S^{2}$ and choosing $f \in \mathcal{H}_{+}\left(S^{1} \times S^{2}\right)$ to be the clutching diffeomorphism giving the twisted $S^{2}$-bundle over $S^{2}, S^{2} \tilde{\times} S^{2}$. Evidently $f_{*} \in A_{L}\left(S^{1} \times S^{2}\right)$ so that $f$ extends to some morphism $(f, \Lambda): B^{2} \times S^{2} \rightarrow B^{2} \times S^{2}$. If $f$ were to extend to a homeomorphism of $B^{2} \times S^{2}$, then it is not hard to argue that $S^{2} \tilde{\times} S^{2}=S^{2} \times S^{2}$. As this is certainly false, there is no such extension.

Define $V=V_{1} \cup_{f}\left(-V_{2}\right)$ and note that $V$ is a closed, 1-connected 4-manifold which, by Novikov additivity (Proposition (7.1) of $[\mathbf{A}, \mathbf{S}]$ ), has signature zero. Further, if $\Delta(V) \in H^{4}(V ; \mathbf{Z} / 2) \cong \mathbf{Z} / 2$ is the Kirby-Siebenmann obstruction to smoothing $V$, then

$$
\Delta(V)=\Delta\left(V_{1}\right)+\Delta\left(V_{2}\right)
$$

where $\Delta\left(V_{1}\right)$ are $\Delta\left(V_{2}\right)$ are defined as in the introduction.

The commutative diagram, Diagram (IV.2) below, names most of the homomorphisms used in this section. It is based on the following diagram of inclusions:

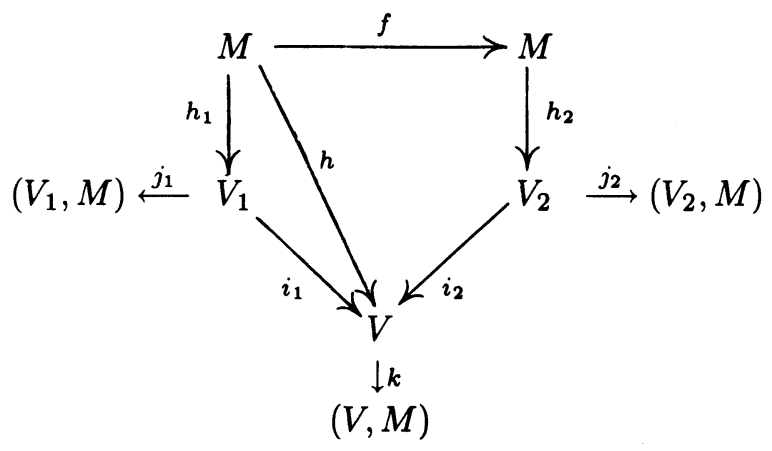

The central column and both rows of (IV.2) are exact. 


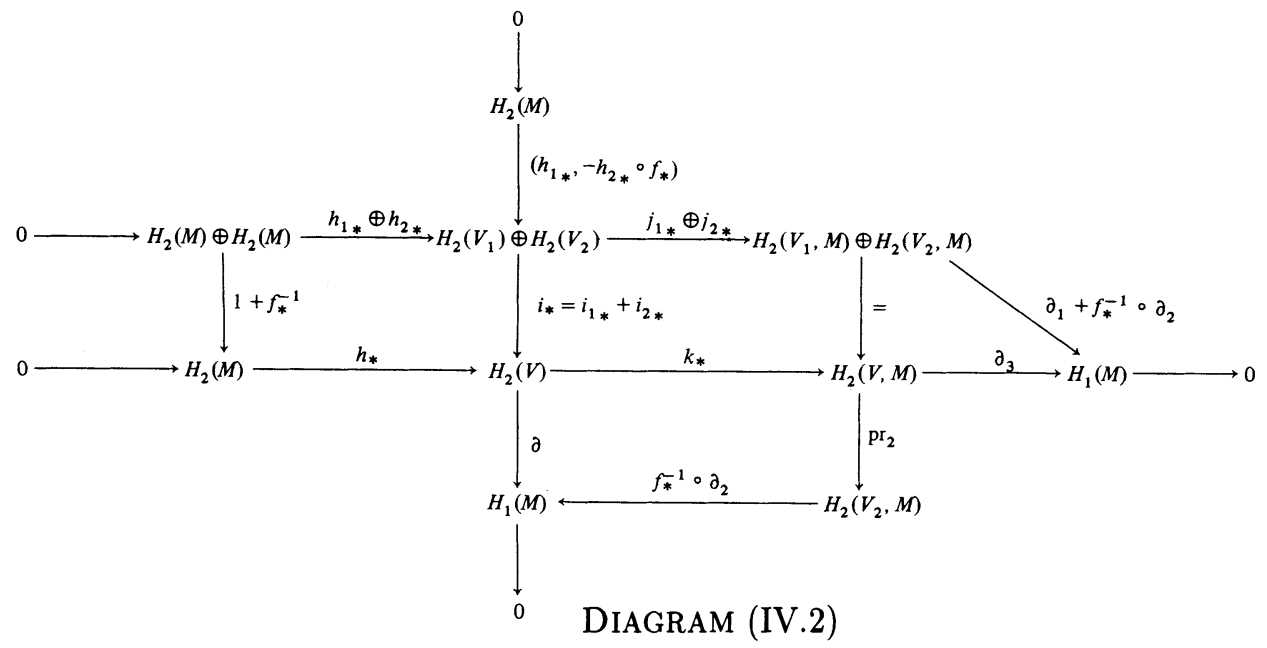

Finally, for any homomorphism $\Psi: A \rightarrow B$ of abelian groups, $G(\Psi)$ will be the subgroup of $A \oplus B$ corresponding to the graph of $\Psi$ :

$$
G(\Psi)=\{(a, \Psi(a)) \mid a \in A\}
$$

(4.2) Proposition. The morphism $(f, \Lambda)$ is realized geometrically by a homeomorphism $F: V_{1} \rightarrow V_{2}$ if and only if $\Delta\left(V_{1}\right) \equiv \Delta\left(V_{2}\right)$ and there is a maximal isotropic subgroup $J \subseteq H_{2}(V)$ such that

(i) $i_{*}(G(-\Lambda)) \subseteq J$;

(ii) $\partial(J)=H_{1}(M)$.

Proof. If $f$ extends to a homeomorphism $F: V_{1} \rightarrow V_{2}$ realizing $\Lambda$, then clearly $\Delta\left(V_{1}\right) \equiv \Delta\left(V_{2}\right)$.

For the rest let $J=\operatorname{image}\left(H_{2}\left(V_{1}, M\right) \rightarrow H_{2}(V)\right)$ under the "doubling" homeomorphism given by $F$. This homeomorphism sends the class of a relative cycle $z \in Z_{2}\left(V_{1}, M\right)$ to the class in $H_{2}(V)$ represented by the absolute cycle $z-F_{\#}(z)$. One readily verifies that $J$ has the desired properties.

On the other hand, assume that $\Delta\left(V_{1}\right) \equiv \Delta\left(V_{2}\right)$ and that there is a subgroup $J \subseteq H_{2}(V)$ as in the hypotheses. Now from equation (IV.1) we have $\Delta(V) \equiv 0$, and therefore $V$ is a stably smoothable, 1-connected 4-manifold with signature zero. Using Freedman's classification theorem (Theorem (1.5) of [Fr] and Corollary (2.2.3) of $[\mathbf{Q}])$, we conclude there is an $n \geq 0$ such that

$$
V \cong \#_{i=1}^{n} S^{2} \times S^{2} \quad \text { or } \quad V=\left(\prod_{i=1}^{n-1} S^{2} \times S^{2}\right) \#\left(S^{2} \tilde{\times} S^{2}\right) .
$$

In either instance, $V$ bounds a 5 -manifold $W$ which is the boundary connected sum of $n D^{3}$-bundles over $S^{2}$. Note that $W \simeq \bigvee_{i=1}^{n} S^{2}$. As in Theorem (2) of [W $\mathbf{1}$, we may replace $W$ by another 5 -manifold $W_{1}$ with $\partial W_{1}=V, W_{1} \simeq \bigvee_{i=1}^{n} S^{2}$ and $J=\operatorname{ker}\left(H_{2}(V) \rightarrow H_{2}\left(W_{1}\right)\right)$. We shall show that the triple $\left(W_{1} ; V_{1}, V_{2}\right)$ is a relative $h$-cobordism. Thus by the 5 -dimensional relative $h$-cobordism theorem (Theorem (2.1.1) of $[\mathbf{Q}]), f$ extends to a homeomorphism $F: V_{1} \rightarrow V_{2}$.

Now $V_{1} \simeq V_{2} \simeq W_{1} \simeq \bigvee_{i=1}^{n} S^{2}$ so to prove that the inclusion $V_{i} \rightarrow W$ is a homotopy equivalence it suffices to show that $H_{2}\left(V_{i}\right) \rightarrow H_{2}\left(W_{1}\right)(i=1,2)$. To that 
end consider the commutative diagram

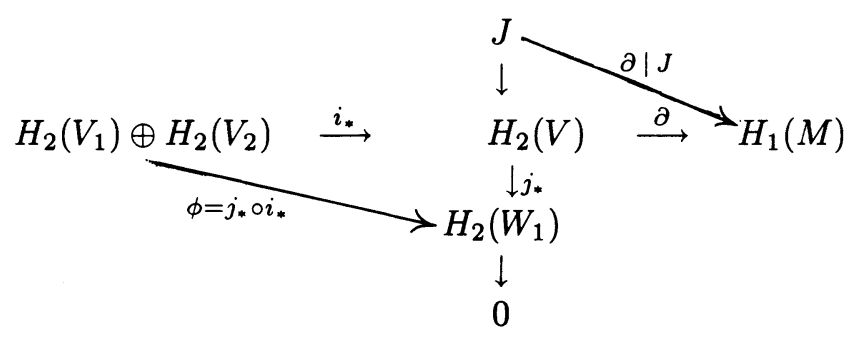

Both row and column are exact.

Now by assumption, $\partial \mid J$ is surjective and a quick diagram chase shows that $\phi$ is also. Thus if $\xi \in H_{2}\left(W_{1}\right)$, there are elements $\xi_{i} \in H_{2}\left(V_{i}\right)(i=1,2)$ such that $\xi=\phi\left(\xi_{1}, \xi_{2}\right)$. But as $i_{*}(G(-\Lambda)) \subseteq J=\operatorname{Ker}\left(j_{*}\right)$

$$
\phi\left(-\xi_{1}, \Lambda\left(\xi_{1}\right)\right)=\phi\left(\Lambda^{-1}\left(\xi_{2}\right),-\xi_{2}\right)=0 .
$$

Thus

$$
\begin{aligned}
\xi & =\phi\left(\xi_{1}, \xi_{2}\right) \\
& =\left\{\begin{array}{l}
\phi\left(\xi_{1}, \xi_{2}\right)+\phi\left(-\xi_{1}, \Lambda\left(\xi_{1}\right)\right) \\
\phi\left(\xi_{1}, \xi_{2}\right)+\phi\left(\Lambda^{-1}\left(\xi_{2}\right),-\xi_{2}\right)
\end{array}\right. \\
& =\left\{\begin{array}{l}
\phi\left(0, \xi_{2}+\Lambda\left(\xi_{1}\right)\right) \\
\phi\left(\xi_{1}+\Lambda^{-1}\left(\xi_{2}\right), 0\right)
\end{array}\right.
\end{aligned}
$$

Clearly this implies that $\xi \in \operatorname{image}\left(H_{2}\left(V_{i}\right) \rightarrow H_{2}\left(W_{1}\right)\right)(i=1,2)$, and as $\xi$ was chosen arbitrarily, both homomorphisms $H_{2}\left(V_{i}\right) \rightarrow H_{2}\left(W_{1}\right)$ are surjective. As noted above, this means that $f$ extends to a homeomorphism $F: V_{1} \rightarrow V_{2}$.

Finally, to see that $F_{*}=\Lambda$, let $\phi_{i}=\phi \mid H_{2}\left(V_{i}\right)(i=1,2)$. Evidently $F_{*}=\phi_{2}^{-1} \circ \phi_{1}$, which, as $G(-\Lambda) \subseteq \operatorname{ker}(\phi)$, is precisely $\Lambda$.

The proof is now complete.

Our goal now is to translate the conditions of Proposition (4.2) into the ones occurring in the statement of Theorem (0.7). Before doing this, we need a reformulation of the invariant $\theta(f, \Lambda)$ in the terms of homology data from the manifold $V$.

(4.3) LeMmA. Let $\mu \in H_{2}(V)$ be such that $k_{*}(\mu) \in G\left(-\Lambda^{*}\right)$. Then

$$
\left\langle\theta(f, \Lambda), f_{*} \circ \partial(\mu)\right\rangle \equiv \mu \cdot \mu \quad(\bmod 2) .
$$

ProOF. Let $k^{*}: H^{2}\left(V_{1}, M ; \mathbf{Z} / 2\right) \oplus H^{2}\left(V_{2}, M ; \mathbf{Z} / 2\right) \rightarrow H^{2}(V ; \mathbf{Z} / 2)$ be adjoint to $k_{*}$. Then as $V$ is formed by gluing $V_{1}$ to $V_{2}$ by $f$, if $\sigma \in \operatorname{Spin}(M)$,

$$
w_{2}(V)=k^{*}\left(w_{2}\left(V_{1}, M ; \sigma\right), w_{2}\left(V_{2}, M ; f_{\#}(\sigma)\right)\right)
$$


Hence if $\eta \in H_{2}\left(V_{2}, M\right)$ is chosen so that $k_{*}(\mu)=\left(-\Lambda^{*}(\eta), \eta\right)$,

$$
\begin{aligned}
\mu \cdot \mu & \equiv\left\langle w_{2}(V), \mu\right\rangle \quad \text { by Wu's formula } \\
& \equiv\left\langle k^{*}\left(w_{2}\left(V_{1}, M ; \sigma\right), w_{2}\left(V_{2}, M ; f_{\#}(\sigma)\right)\right), \mu\right\rangle \\
& \equiv\left\langle\left(w_{2}\left(V_{1}, M ; \sigma\right), w_{2}\left(V_{2}, M ; f_{\#}(\sigma)\right)\right),\left(-\Lambda^{*}(\eta), \eta\right)\right\rangle \\
& \equiv\left\langle w_{2}\left(V_{1}, M ; \sigma\right),-\Lambda^{*}(\eta)\right\rangle+\left\langle w_{2}\left(V_{2}, M ; f_{\#}(\sigma)\right), \eta\right\rangle \\
& \equiv\left\langle w_{2}\left(V_{1}, M ; \pi_{\Lambda}(\sigma)\right),-\eta\right\rangle+\left\langle w_{2}\left(V_{2}, M ; f_{\#}(\sigma)\right), \eta\right\rangle \quad \text { by Lemma }(2.9)(\mathrm{i}) \\
& \equiv\left\langle\delta_{2} \theta(f, \Lambda), \eta\right\rangle \quad \text { by Lemma }(4.1)(\mathrm{i}) \\
& \equiv\left\langle\theta(f, \Lambda), \partial_{2}(\eta)\right\rangle \quad \\
& \equiv\left\langle\theta(f, \Lambda), f_{*} \circ \partial(\mu)\right\rangle \quad \text { see Diagram (IV.2). } \quad \square
\end{aligned}
$$

Proof of Theorem (0.7). Assume first of all that the morphism $(f, \Lambda)$ is realized geometrically by a homeomorphism $F: V_{1} \rightarrow V_{2}$. Clearly $\Delta\left(V_{1}\right) \equiv \Delta\left(V_{2}\right)$. To show that $\theta(f, \Lambda) \equiv 0$, it suffices to prove that $f_{\#}=\pi_{F_{*}}$. To that end let $\sigma \in \operatorname{Spin}(M)$. Using the definition of the functions $w_{2}\left(V_{i}, M\right)(i=1,2)$ it can be shown that $w_{2}\left(V_{2}, M ; f_{\#}(\sigma)\right)=\left(F^{*}\right)^{-1} w_{2}\left(V_{1}, M ; \sigma\right)$. Hence,

$$
\begin{aligned}
\pi_{F_{*}}(\sigma) & =\gamma_{2}^{-1} \circ F_{*} \circ \gamma_{1}(\sigma) \\
& =\gamma_{2}^{-1} \circ F_{*} \circ D_{V_{1}}\left(w_{2}\left(V_{1}, M ; \sigma\right)\right) \\
& =\gamma_{2}^{-1} \circ D_{V_{2}} \circ\left(F^{*}\right)^{-1}\left(w_{2}\left(V_{1}, M ; \sigma\right)\right) \quad \text { as } F \text { has degree } 1, \\
& =\gamma_{2}^{-1} \circ D_{V_{2}}\left(w_{2}\left(V_{2}, M ; f_{\#}(\sigma)\right)\right) \\
& =f_{\#}(\sigma) \text { by the identity (II.1). }
\end{aligned}
$$

Thus $f_{\#}=\pi_{F *}$ and so $\theta(f, \Lambda) \equiv 0$ as claimed.

To prove the converse we shall show that the hypothesis $\theta(f, \Lambda) \equiv 0$ implies there is a maximal isotropic subgroup $J$ of $H_{2}(V)$ such that $i_{*}(G(-\Lambda)) \subseteq J$ and $\partial(J)=H_{1}(M)$. The desired conclusion will then follow from Proposition (4.2).

The subgroup $J$ will be built as the sum of two isotropic subgroups $J_{1}$ and $J_{2}$ of $H_{2}(V)$ which satisfy

(i) $i_{*}(G(-\Lambda)) \subset J_{1}, \partial\left(J_{1}\right)=T_{1}(M)$ and

$$
\operatorname{rank}\left(J_{1}\right)=\operatorname{rank}\left(H_{2}\left(V_{1}\right)\right)-\operatorname{rank}\left(H_{1}(M)\right) ;
$$

(ii) the composition $J_{2} \stackrel{\partial \mid J_{2}}{\longrightarrow} H_{1}(M) \rightarrow H_{1}(M) / T_{1}(M)$ is an isomorphism;

(iii) $J_{1} \cap J_{2}=\{0\}$ and $J_{1} \cdot J_{2}=\{0\}$.

Assuming we have found such subgroups, we let $J$ be the smallest direct summand of $H_{2}(V)$ containing $J_{1}+J_{2}$. As $\operatorname{rank}\left(H_{2}(V)\right)=2 \operatorname{rank}\left(H_{2}\left(V_{1}\right)\right), J$ is evidently the desired subgroup of $H_{2}(V)$ and the proof will be complete.

CONSTRUCTION OF $J_{1}$. Let $J_{1}$ be the smallest direct summand of $H_{2}(V)$ containing $i_{*}(G(-\Lambda))$. Now $i_{*}(G(-\Lambda))$ is isotropic in $H_{2}(V)$, as $\Lambda$ is an isometry. Thus $J_{1}$ is also.

Next we prove $\partial\left(J_{1}\right)=T_{1}(M)$. If $\mu \in J_{1}$, there is an integer $m>0$ such that $m \mu \in i_{*}(G(-\Lambda))$. But then $m \partial(\mu)=0$, as $\partial \circ i_{*}=0$. Hence $\partial(\mu) \in T_{1}(M)$ which shows $\partial\left(J_{1}\right) \subseteq T_{1}(M)$.

To derive the opposite inclusion, let $\nu \in T_{1}(M)$. 
(4.4) LEMMA. There is a class $\mu \in H_{2}(V)$ with $\partial(\mu)=\nu$ and $k_{*}(\mu) \in G\left(-\Lambda^{*}\right)$.

Proof. Fix any $\eta \in H_{2}\left(V_{2}, M\right)$ such that $\partial_{2}(\eta)=f_{*}(\nu)$. Now as $(f, \Lambda)$ is a morphism, $G\left(-\Lambda^{*}\right) \subset \operatorname{image}\left(k_{*}\right)$ (see Diagram (IV.2)), so we may choose a $\mu \in H_{2}(V)$ for which $k_{*}(\mu)=\left(-\Lambda^{*}(\eta), \eta\right)$. Further,

$$
\begin{aligned}
\partial(\mu) & =f_{*}^{-1} \circ \partial_{2} \circ \mathrm{pr}_{2} \circ k_{*}(\mu) \quad \text { see Diagram (IV.2) } \\
& =f_{*}^{-1} \circ \partial_{2}(\eta) \\
& =\nu \quad \text { by choice of } \eta . \quad \square
\end{aligned}
$$

Choose $\mu$ as in the lemma and set $k_{*}(\mu)=\left(-\Lambda^{*}(\eta), \eta\right)$. Now since $\nu \in T_{1}(M)$, we may find an $m>0$ and $\xi \in H_{2}\left(V_{1}\right)$ such that $j_{1^{*}}(\xi)=-m \Lambda^{*}(\eta)$. Then $k_{*} \circ i_{*}(\xi,-\Lambda(\xi))=m\left(-\Lambda^{*}(\eta), \eta\right)$ (see Diagram (IV.2)) and thus

$$
i_{*}\left(\xi,-\Lambda^{*}(\xi)\right)=m \mu+h_{*}(\beta)
$$

for some $\beta \in H_{2}(M)$.

(4.5) Lemma. The class $\beta$ is divisible by $m$ in $H_{2}(M)$.

We delay the demonstration of this lemma till the end of the proof of Theorem (0.7). Assuming it, we see from equation (IV.3) that $\mu+h_{*}(\beta / m) \in J_{1}$. Then

$$
\nu=\partial(\mu)=\partial\left(\mu+h_{*}(\beta / m)\right) .
$$

Thus $\nu \in \partial\left(J_{1}\right)$ and as $\nu$ was chosen arbitrarily, we conclude $\partial\left(J_{1}\right)=T_{1}(M)$.

Finally, to calculate $\operatorname{rank}\left(J_{1}\right)$, note

$$
\operatorname{ker}\left(i_{*}\right)=\left\{\left(h_{1_{*}}(\beta),-h_{2 .} f_{*}(\beta)\right) \mid \beta \in H_{2}(M)\right\} \subseteq G(-\Lambda) .
$$

Thus

$$
\begin{aligned}
\operatorname{rank}\left(J_{1}\right) & =\operatorname{rank}\left(i_{*}(G(-\Lambda))\right)=\operatorname{rank}(G(-\Lambda))-\operatorname{rank}\left(\operatorname{ker}\left(i_{*}\right)\right) \\
& =\operatorname{rank}\left(H_{2}\left(V_{1}\right)\right)-\operatorname{rank}\left(H_{1}(M)\right) .
\end{aligned}
$$

CONSTRUCTION OF $J_{2}$. Set $F_{1}(M)=H_{1}(M) / T_{1}(M)$ and choose $\nu_{1}, \nu_{2}, \ldots, \nu_{m}$ $\in H_{1}(M)$ which project to a basis of this group. By Lemma (4.4), there are classes $\mu_{1}^{\prime}, \mu_{2}^{\prime}, \ldots \mu_{m}^{\prime} \in H_{2}(V)$ such that

(i) $\partial\left(\mu_{i}^{\prime}\right)=\nu_{i}, 1 \leq i \leq m$

(ii) $k_{*}\left(\mu_{i}^{\prime}\right) \in G\left(-\Lambda^{*}\right), 1 \leq i \leq m$.

Let $\beta_{1}, \beta_{2}, \ldots, \beta_{m} \in H_{2}(M)$ be the basis dual to $\nu_{1}, \ldots, \nu_{m}$. That is $\beta_{i} \cdot \nu_{j}=\delta_{i j}$. Set $\bar{\beta}_{i}=h_{*}\left(\beta_{i}\right)(1 \leq i \leq m)$ and note that

(i) $\overline{\beta_{i}} \cdot \overline{\beta_{j}}=0,1 \leq i, j \leq m$;

(ii) $\overline{\beta_{i}} \cdot \mu_{j}^{\prime}=\delta_{i j}, 1 \leq i, j \leq m$.

Define

$$
\mu_{i}^{\prime \prime}=\mu_{i}^{\prime}-\sum_{j=i+1}^{m}\left(\mu_{i}^{\prime} \cdot \mu_{j}^{\prime}\right) \overline{\beta_{j}}, \quad 1 \leq i \leq m,
$$

and observe that $k_{*}\left(\mu_{i}^{\prime \prime}\right)=k_{*}\left(\mu_{i}^{\prime}\right) \in G\left(-\Lambda^{*}\right)$. Thus for each $i$,

$$
\begin{aligned}
\mu_{i}^{\prime \prime} \cdot \mu_{i}^{\prime \prime} & \equiv \theta(f, \Lambda)\left(f_{*} \circ \partial\left(\mu_{i}^{\prime \prime}\right)\right) \quad \text { by Lemma }(4.3) \\
& \equiv 0(\bmod 2) \quad \text { as } \theta(f, \Lambda) \equiv 0 .
\end{aligned}
$$

Thus we may form

$$
\mu_{i}=\mu_{i}^{\prime \prime}-\frac{1}{2}\left(\mu_{i}^{\prime \prime} \cdot \mu_{i}^{\prime \prime}\right) \overline{\beta_{i}} \in H_{2}(V), \quad 1 \leq i \leq m .
$$


Now it can be checked that $\mu_{i} \cdot \mu_{j}=0(1 \leq i, j \leq m), \partial\left(\mu_{i}\right)=\nu_{i}(1 \leq i \leq m)$, and $k_{*}\left(\mu_{i}\right) \in G\left(-\Lambda^{*}\right)$. Thus if we set $J_{2}=\operatorname{Span}\left(\mu_{1}, \mu_{2}, \ldots, \mu_{m}\right) \subseteq H_{2}(V)$,

(i) $J_{2}$ is isotropic;

(ii) the composition $J_{2} \stackrel{\partial \mid J_{2}}{\longrightarrow} H_{1}(M) \rightarrow F_{1}(M)$ is an isomorphism;

(iii) $k_{*}\left(J_{2}\right) \subseteq G\left(-\Lambda^{*}\right)$.

Thus $J_{2}$ satisfies the desired properties.

PROOF THAT $J_{1} \cap J_{2}=0$ AND $J_{1} \cdot J_{2}=0$. That $J_{1} \cap J_{2}=0$, simply observe that under the composition

$$
H_{2}(V) \stackrel{\partial}{\rightarrow} H_{1}(M) \rightarrow F_{1}(M)
$$

$J_{1}$ maps to zero while $J_{2}$ maps monomorphically.

To see that $J_{1} \cdot J_{2}=0$, choose $\mu_{i} \in J_{i}(i=1,2)$. Now by the construction of $J_{1}$ and $J_{2}$, we may choose an integer $m>0$, and elements $\xi \in H_{2}\left(V_{1}\right)$ and $\eta \in H_{2}\left(V_{2}, M\right)$ such that $m \mu_{1}=i_{*}(\xi,-\Lambda(\xi)), k_{*}\left(\mu_{2}\right)=\left(-\Lambda^{*}(\eta), \eta\right)$. Then

$$
\mu_{1} \cdot \mu_{2}=\frac{1}{m} i_{*}(\xi,-\Lambda(\xi)) \cdot \mu_{2}=\frac{1}{m}\left[\xi \cdot\left(-\Lambda^{*}(\eta)\right)+\Lambda(\xi) \cdot \eta\right]=0 .
$$

As $\mu_{1}, \mu_{2}$ were arbitrary, $J_{1} \cdot J_{2}=0$.

To complete the demonstration of Theorem (0.7), we must prove Lemma (4.5).

PROOF OF LEMMA (4.5). It suffices to show that $\beta \cdot \nu \equiv 0(\bmod m)$ for each $\nu \in H_{1}(M)$. But from the properties of $J_{2}$, for any such $\nu$ there is some $\mu^{\prime} \in J_{2}$ with $\partial\left(\mu^{\prime}\right)-\nu \in T_{1}(M)$. Then

$$
\begin{aligned}
\beta \cdot \nu & =h_{*}(\beta) \cdot \mu^{\prime}=i_{*}(\xi,-\Lambda(\xi)) \cdot \mu^{\prime}-m \mu \cdot \mu^{\prime} \quad \text { by }(\mathrm{IV} .3) \\
& =-m \mu \cdot \mu^{\prime}
\end{aligned}
$$

as $i_{*}(\xi,-\Lambda(\xi)) \in J_{1}$. Thus Lemma (4.5) follows.

PROOF OF THEOREM (0.10). According to Corollary (1.8), there is an integer $m_{1}>0$ depending only on $T_{1}(M)$ such that for any 1-connected 4-manifold $V$ with boundary $M$ and $f \in H_{+}(M)$ there is a morphism $\left(f^{m_{1}}, \Lambda\right): V \rightarrow V$.

According to Proposition (4.1)(iv), there is an $m_{2}>0$ depending only on $H_{1}(M)$ such that $\theta\left(f^{m_{1} m_{2}}, \Lambda^{m_{2}}\right)=0$. By Theorem $(0.7), f^{m_{1} m_{2}}$ extends to a homeomorphism $F: V \rightarrow V$.

We complete this section by proving Proposition (0.8).

ProOF OF PROPOSITION (0.8). (i) When $H_{1}(M ; \mathbf{Q}) \cong 0, I^{1}(M) \cong 0$. Thus as $\theta(f, \Lambda) \in I^{1}(M), \theta(f, \Lambda)=0$.

(ii) This statement requires more work. First note that if $\phi: H_{2}\left(V_{1}, M\right) \rightarrow$ $H_{2}(M)$ is an arbitrary homomorphism then setting $\Lambda^{\prime}=\Lambda+h_{2 *} \circ \phi \circ j_{1 *},\left(f, \Lambda^{\prime}\right): V_{1} \rightarrow$ $V_{2}$ is a morphism. To calculate $\theta\left(f, \Lambda^{\prime}\right)$, set

$$
y(\phi)=D_{M}^{-1} \circ \phi \circ D_{V_{1}}\left(w_{2}\left(V_{1}\right)\right) \in I^{1}(M) .
$$

Now by the definition of $\gamma_{1}: \operatorname{Spin}(M) \rightarrow H_{2}\left(V_{1} ; \mathbf{Z} / 2\right)(\S 2)$ and Lemma (2.7)(i), it can be shown that $j_{1 *}\left(\gamma_{1}(\sigma)\right)=D_{V_{1}}\left(w_{2}\left(V_{1}\right)\right)$ for any $\sigma \in \operatorname{Spin}(M)$. Then

$$
\begin{aligned}
\pi_{\Lambda^{\prime}}(\sigma) & =\gamma_{2}^{-1}\left(\Lambda\left(\gamma_{1}(\sigma)\right)+h_{2 *} \circ \phi \circ j_{1 *}\left(\gamma_{1}(\sigma)\right)\right) \\
& =\gamma_{2}^{-1}\left(\gamma_{2}\left(\pi_{\Lambda}(\sigma)\right)+h_{2 *} \circ D_{M}(y(\phi))\right) \quad \text { by Lemma }(2.9)(\mathrm{i}) \\
& =\gamma_{2}^{-1}\left(\gamma_{2}\left(y(\phi) \cdot \pi_{\Lambda}(\sigma)\right)\right) \quad \text { by Lemma }(2.8)(\mathrm{ii}) \\
& =y(\phi) \cdot \pi_{\Lambda}(\sigma) .
\end{aligned}
$$

Hence $\theta\left(f, \Lambda^{\prime}\right)=\theta(f, \Lambda)+y(\phi)$. 
Next choose a basis $\xi_{1}, \xi_{2}, \ldots, \xi_{n}$ for $H_{2}\left(V_{1}\right)$ with $\xi_{1}^{*}, \xi_{2}^{*}, \ldots, \xi_{n}^{*}$ the associated dual basis of $H_{2}\left(V_{1}, M\right)$. The Wu formula implies that

$$
D_{V_{1}}\left(w_{2}\left(V_{1}\right)\right) \equiv \sum_{i=1}^{n}\left(\xi_{i} \cdot \xi_{i}\right) \xi_{i}^{*} \quad(\bmod 2)
$$

Hence

$$
y(\phi)=\sum_{i=1}^{n}\left(\xi_{i} \cdot \xi_{i}\right) D_{M}^{-1} \phi\left(\xi_{i}^{*}\right) .
$$

Now suppose we have chosen the basis $\xi_{1}, \ldots, \xi_{n}$ so that $\xi_{i} \cdot \xi_{i} \equiv 1(\bmod 2)$ if and only if $i=1$. Then $y(\phi) \equiv D_{M}^{-1} \phi\left(\xi_{1}^{*}\right)$. Hence if $y \in I^{1}(M)$ is given, choose any $\phi$ so that $D_{M}^{-1} \cdot \phi\left(\xi_{1}^{*}\right)=y$. The associated morphism $\left(f, \Lambda^{\prime}\right)$ has $\theta\left(f, \Lambda^{\prime}\right)=\theta(f, \Lambda)+y$. Evidently this suffices to deduce (ii).

(iii) That $\theta(f, \Lambda)$ is independent of $\Lambda$ and is given by $d\left(f_{\#}\left(\sigma_{V_{1}}\right), \sigma_{V_{2}}\right)$ is precisely Proposition $(4.1)(\mathrm{v})$. As $V$ admits a spin structure if and only if $f_{\#}\left(\sigma_{V_{1}}\right)=\sigma_{V_{2}}$, we are done.

5. In this final section we prove Theorems $(0.1)$ and $(0.2)$ as well as Propositions (0.5) and (0.6).

Fix a bilinear form space $\left(\mathbf{Z}^{n}, L\right)$ which presents $H_{*}(M)$ and suppose $V$ is a 1connected 4-manifold with boundary $M$ and $\Lambda:\left(\mathbf{Z}^{n}, L\right) \rightarrow\left(H_{2}(V), \cdot\right)$ an isometry.

(5.1) LemMA. (i) The double coset $H_{+}(M) \partial(\Lambda) A_{L}(M) \in B_{L}(M)$ depends only upon the class of $V$ in $V_{L}(M)$.

(ii) If $L$ is even, the double coset $\hat{H}_{+}(M)\left(\partial(\Lambda), \pi_{\Lambda}\right) \hat{A}_{L}(M)$ depends only upon the class of $V$.

ProOF. Let $V_{1}$ be another 1-connected 4-manifold with boundary $M$ and suppose there is a homeomorphism $F: V \rightarrow V_{1}$. Let $\Psi:\left(\mathbf{Z}^{n}, L\right) \rightarrow\left(H_{2}\left(V_{1}\right), \cdot\right)$ be an arbitrary isometry. Then with $f=F \mid M$,

$$
\begin{aligned}
H_{+}(M) \partial(\Psi) A_{L}(M) & =H_{+}(M) f_{*} \circ \partial(\Lambda) \circ \partial\left(\Lambda^{-1} \circ F_{*}^{-1} \circ \Psi\right) A_{L}(M) \\
& =H_{+}(M) \partial(\Lambda) A_{L}(M) .
\end{aligned}
$$

This proves the first part of the lemma.

To prove the second part, note that Theorem (0.7) shows $\theta\left(f, F_{*}\right)=0$. Hence $f_{\#}=\pi_{F}$, and therefore

$$
\left(\partial(\Psi), \pi_{\Psi}\right)=\left(f_{*}, f_{\#}\right) \circ\left(\partial(\Lambda), \pi_{\Lambda}\right) \circ\left(\partial\left(\Lambda^{-1} \circ F_{*}^{-1} \circ \Psi\right), \pi_{\Lambda^{-1} \circ F_{*}^{-1} \circ \Psi}\right) .
$$

This equality implies the desired result.

We shall denote the class of $V$ in $\mathcal{V}_{L}(M)$ by $[V]$. Lemma (5.1) shows that there is a well-defined function

$$
c_{L}: \mathcal{V}_{L}(M) \rightarrow B_{L}(M), \quad[V] \rightarrow H_{+}(M) \partial(\Lambda) A_{L}(M),
$$

where $\Lambda:\left(\mathbf{Z}^{n}, L\right) \rightarrow\left(H_{2}(V), \cdot\right)$ is an isometry.

Similarly when $L$ is even there is a function

$$
\hat{c}_{L}: V_{L}(M) \rightarrow \hat{B}_{L}(M), \quad[V] \rightarrow \hat{H}_{+}(M)\left(\partial(\Lambda), \pi_{\Lambda}\right) \hat{A}_{L}(M) .
$$

Evidently if $p_{1}: \hat{B}_{L}(M) \rightarrow B_{L}(M)$ is the natural projection (see $\S 3$ ), then $p_{1} \circ \hat{c}_{L}=$ $c_{L}$. 
(5.2) LEMMA. (i) If $V_{1}$ and $V_{2}$ represent classes in $V_{L}(M)$, then $c_{L}\left(\left[V_{1}\right]\right)=$ $c_{L}\left(\left[V_{2}\right]\right)$ if and only if there is a morphism $(f, \Lambda): V_{1} \rightarrow V_{2}$.

(ii) If $L$ is even, then $\hat{c}_{L}\left(\left[V_{1}\right]\right)=\hat{c}_{L}\left(\left[V_{2}\right]\right)$ if and only if there is a morphism $(f, \Lambda): V_{1} \rightarrow V_{2}$ with $\theta(f, \Lambda)=0$.

Proof. Choose isometries $\Psi_{i}:\left(\mathbf{Z}^{n}, L\right) \rightarrow\left(H_{2}\left(V_{i}\right), \cdot\right)(i=1,2)$.

If $c_{L}\left(\left[V_{1}\right]\right)=c_{L}\left(\left[V_{2}\right]\right)$, then there is some $f \in \mathscr{H}_{+}(M)$ and an isometry $\Gamma$ of $\left(\mathbf{Z}^{n}, L\right)$ such that

$$
\partial\left(\Psi_{2}\right)=f_{*} \circ \partial\left(\Psi_{1}\right) \circ \partial(\Gamma) .
$$

The pair $\left(f, \Psi_{2} \circ \Gamma^{-1} \circ \Psi_{1}^{-1}\right)$ is the desired morphism between $V_{1}$ and $V_{2}$.

Conversely, if $(f, \Lambda): V_{1} \rightarrow V_{2}$ is a morphism, then

$$
\partial\left(\Psi_{2}\right)=f_{*} \circ \partial\left(\Psi_{1}\right) \circ \partial\left(\Psi_{1}^{-1} \circ \Lambda^{-1} \circ \Psi_{2}\right),
$$

from which it is apparent that $c_{L}\left(\left[V_{1}\right]\right)=c_{L}\left(\left[V_{2}\right]\right)$. This proves the first part of Lemma (5.2).

The second part follows by an argument essentially the same as that above so we omit it.

Proof of TheOREMS (0.1) AND (0.2). Define $c_{L}^{t}$ to be the composite

$$
\mathcal{V}_{L}(M) \stackrel{c_{L}}{\rightarrow} B_{L}(M) \stackrel{t_{2}}{\rightarrow} B_{L}^{t}(M)
$$

(see $\S 1$ for the definition of $t_{2}$ ). Combining Corollary (1.10), Lemma (5.2) and Corollary (0.9) shows that when $L$ is odd,

$$
C_{L}^{t}=c_{L}^{t} \times \Delta: \mathcal{V}_{L}(M) \rightarrow B_{L}^{t}(M) \times \mathbf{Z} / 2
$$

is injective. This proves Theorem (0.1).

Now assume that $L$ is even. Combining Lemma (5.2) with Theorem (0.7) shows that $\hat{c}_{L}$ is injective.

Finally, let $p_{2}$ denote the composite

$$
\hat{B}_{L}(M) \stackrel{p_{1}}{\rightarrow} B_{1}(M) \stackrel{t_{1}}{\rightarrow} B_{L}^{t}(M) .
$$

By Corollary (1.10), $t_{2}$ is bijective. Thus the desired bijection between $p_{2}^{-1}\left(p_{2}(\langle\alpha, \pi\rangle)\right)$ and $\pi\left(\operatorname{Spin}_{L}(M)\right) / \not_{+}(M, \alpha)$ is assured by Proposition (3.4). This completes the proof of Theorem (0.2).

Proof of Proposition (0.6). That $\left|B_{L}^{t}(M)\right|=1$ is Corollaries (1.9) and (1.10).

Now assume $L$ is even. As $\left|B_{L}^{t}(M)\right|=1$, Theorem (0.2) provides an injection

$$
\psi_{(1,1)}^{-1} \circ \hat{c}_{L}: \mathcal{V}_{L}(M) \rightarrow \operatorname{Spin}_{L}(M) / \not_{+}(M, 1)
$$

But using Proposition (1.6) it is easy to check that $\nvdash_{+}(M, 1)=\not_{+}(M)$. The result follows.

We close the paper by working out several instances in which both $C_{L}^{t}$ and $\hat{c}_{L}$ are bijections.

We start by detailing a construction which will be used in both situations.

Suppose $A$ is an integrally framed link in $M$ such that surgery along $A$ yields a homology 3-sphere $\Sigma(A)$. If $W(A)$ is the contractible 4-manifold with boundary $\Sigma(A)$, we may construct a 1 -connected 4 -manifold by setting

$$
V(A)=(M \times I) \cup_{A}\left(\bigcup_{i=1}^{|A|} H^{(2)}\right) \cup_{\Sigma(A)}(-W(A)) .
$$


Thus $V(A)$ is the trace of the surgery along $A$ together with $W(A)$.

Note that $\partial V(A)=M$ and that by turning the 2-handles upside-down, $V(A) \simeq$ $\bigvee_{i=1}^{|A|} S^{2}$.

We also remark that

$$
\Delta(V(A)) \equiv \Delta(W(A)) \equiv \mu(\Sigma(A)) \quad(\bmod 2) .
$$

Proof of Proposition (0.5). Suppose $H^{1}(M) \cong \mathbf{Z}^{n}$. We shall concentrate first of all on constructing 4-manifolds with form $\left(\mathbf{Z}^{n}, 0\right)$.

Let $K=K_{1} \cup K_{2} \cup \cdots \cup K_{n}$ be a (smoothly) embedded link in $M$ such that the classes of $K_{1}, K_{2}, \ldots, K_{n}$ form a basis for $H_{1}(M)$. Let

$$
\tau=\bigsqcup \tau_{i}: \bigsqcup_{i=1}^{n} S^{1} \times D^{2} \cong T(K)
$$

be a fixed (closed) tubular neighbourhood of $K$ and set $M_{0}=M \backslash \stackrel{\circ}{T}(K)$.

Now using duality and excision, it can be shown that $H_{1}\left(M_{0}\right) \cong \mathbf{Z}^{n}$, while $H_{2}\left(M_{0}\right) \cong \mathbf{Z}^{n-1}$. Plugging these groups into the long exact sequence of the pair $\left(M, M_{0}\right)$ implies that the inclusion $M_{0} \rightarrow M$ induces an isomorphism $H_{1}\left(M_{0}\right) \cong$ $H_{1}(M)$. Hence, there is a basis for $H_{1}\left(M_{0}\right)$ consisting of longitudes of $K_{1}, K_{2}, \ldots$, $K_{n}$. Further, the meridians to these curves are null-homologous in $M_{0}$. Thus we see that any integral surgery along $K$ yields a homology 3 -sphere.

For each $b \in\{0,1\}^{n}$, we may construct an integrally framed link $A_{b}$ whose underlying link is $K$ and whose $i$ th framing curve (see $\S 9 \mathrm{G}$ of [Rlf $]$ ) is given by

$$
\begin{cases}\tau_{i}\left(\left\{(u, *) \mid u \in S^{1},\{*\} \subseteq S^{1}\right\}\right) & \text { if } b(i)=0 \\ \tau_{i}\left(\left\{(u, u) \mid u \in S^{1}\right\}\right) & \text { if } b(i)=1 .\end{cases}
$$

Form $V\left(A_{b}\right)$ as in the discussion prior to the beginning of this proof. As $V\left(A_{b}\right) \simeq$ $\bigvee_{i=1}^{n} S^{2}$ and $H_{1}(M) \cong \mathbf{Z}^{n}$, it is not hard to argue that $\left(H_{2}\left(V\left(A_{b}\right)\right), \cdot\right) \cong\left(\mathbf{Z}^{n}, 0\right)$.

We note that if $\sigma_{b} \in \operatorname{Spin}(M)$ is the unique spin structure extending over $V\left(A_{b}\right)$, then

$$
\operatorname{Spin}(M)=\left\{\sigma_{b} \mid b \in\{0,1\}^{n}\right\} .
$$

To see this suppose $b_{1}, b_{2}$ are distinct elements of $\{0,1\}^{n}$. Then there is an $i \in\{1,2, \ldots, n\}$ for which $\left|b_{1}(i)-b_{2}(i)\right|=1$. But then by the construction of $V\left(A_{b_{j}}\right)(j=1,2), \sigma_{b_{1}}$ will not extend over the $j$ th 2-handle of $V\left(A_{b_{2}}\right)$. Thus $\sigma_{b_{1}} \neq \sigma_{b_{2}}$ and a counting argument shows (V.2) holds.

Now to the proof of Proposition (0.5) proper.

Let $\left(\mathbf{Z}^{m}, L\right)$ be an arbitrary form presenting $H_{*}(M)$. Then $\left(\mathbf{Z}^{m}, L\right)$ splits orthogonally as $\left(\mathbf{Z}^{n}, 0\right) \oplus\left(\mathbf{Z}^{m-n}, L_{1}\right)$ where $\left(\mathbf{Z}^{m-n}, L_{1}\right)$ is a unimodular form which is odd if and only if $\left(\mathbf{Z}^{m}, L\right)$ is.

First assume that $L$ is odd. According to Freedman (Theorem (1.5) of [Fr]), there are 1-connected 4-manifolds $V_{1}^{\prime}$ and $V_{2}^{\prime}$ such that

(i) $\partial\left(V_{1}^{\prime}\right) \cong \partial\left(V_{2}^{\prime}\right) \cong S^{3}$;

(ii) $\left(H_{2}\left(V_{i}^{\prime}\right), \cdot\right) \cong\left(\mathbf{Z}^{m-n}, L_{1}\right)(i=1,2)$;

(iii) $\Delta\left(V_{1}^{\prime}\right) \not \equiv \Delta\left(V_{2}^{\prime}\right)$.

Let $V_{0}$ be any 1-connected 4-manifold with boundary $M$ and intersection form $\left(\mathbf{Z}^{n}, 0\right)$, as constructed above. Then if

$$
V_{i}=V_{0} \downarrow V_{i}^{\prime} \quad(i=1,2)
$$


we have that $\partial\left(V_{i}\right) \cong M,\left(H_{2}\left(V_{i}\right), \cdot\right) \cong\left(\mathbf{Z}^{m}, L\right)$, while $\Delta\left(V_{1}\right) \not \equiv \Delta\left(V_{2}\right)$. Thus $\Delta: V_{L}(M) \rightarrow \mathbf{Z} / 2$ is bijective as claimed.

When $L$ is even, Freedman's work shows there is a unique 4-manifold $V_{1}^{\prime}$ with boundary $S^{3}$ and form $\left(\mathbf{Z}^{m-n}, L_{1}\right)$. Taking the boundary connected sum of $V_{1}^{\prime}$ with the manifolds $V\left(A_{b}\right), b \in\{0,1\}^{n}$, previously constructed, provides us with manifolds $V_{1}(b), b \in\{0,1\}^{n}$, each having boundary $M$ and form $\left(\mathbf{Z}^{m}, L\right)$. Further, by (V.2), $\operatorname{Spin}(M)=\left\{\sigma_{V_{1}\left(A_{b}\right)} \mid b \in\{0,1\}^{n}\right\}$. Finally, from Corollary (1.7) and Theorem $(0.2)$, it now follows that the composition $\psi_{(1,1)}^{-1} \circ \hat{c}_{L}: \mathcal{V}_{L}(M) \rightarrow \operatorname{Spin}(M) / H_{+}(M)$ is a bijection.

This completes the proof of Proposition (0.5).

(5.3) REMARKS. (i) Let $M=\#_{i=1}^{n} S^{1} \times S^{2}, n \geq 0$. It is well known that $H_{+}(M)$ acts transitively on $\operatorname{Spin}(M)$. Thus

$$
\left|\mathcal{V}_{L}\left(\#_{i=1}^{n} S^{1} \times S^{2}\right)\right|= \begin{cases}2, & L \text { is odd } \\ 1, & L \text { is even }\end{cases}
$$

Further, when $L$ is odd, the two manifolds are distinguished by their Kirby-Siebenmann invariants.

(ii) It seems likely that a construction similar to that in the last proposition will show that image $\left(\hat{c}_{L}\right)=p_{1}^{-1}\left(\operatorname{image}\left(c_{L}\right)\right)$ when $L$ is even.

Our next example shows that $C_{L}^{t}$ and $\hat{c}_{L}$ may be bijections when $T_{1}(M) \not\{0\}$.

(5.4) EXAMPLE. Suppose that $H_{*}(M)$ is presented by the form $(\mathbf{Z}, L)$ where $L(n, m)=r n m, r \neq 0$. We shall show that when $r$ is odd, $C_{L}^{t}: \mathcal{V}_{L}(M) \rightarrow B_{L}^{t}(M) \times$ $\mathbf{Z} / 2$ is bijective, as $c_{L}^{t}: \mathcal{V}_{L}(M) \rightarrow B_{L}^{t}(M)$ is when $r$ is even.

Now a quick calculation shows that $H_{1}(M)=T_{1}(M) \cong \mathbf{Z} / r$. Evidently then,

$$
A(M)=\left\{u \mid u^{2} \equiv 1\right\} \subseteq \mathbf{Z} / r
$$

Note that $A(M)$ is abelian so that

$$
B_{L}(M)=A(M) /\left[H_{+}(M) A_{L}(M)\right]
$$

inherits a transitive action from $A(M)$ :

$$
u\left\langle u_{1}\right\rangle=\left\langle u u_{1}\right\rangle \text {. }
$$

We remark that $A_{L}(M)=\{ \pm 1\}$, but $H_{+}(M)$ can be an arbitrary subgroup of $A(M)$, depending on $M$.

We state the following lemma without proof.

(5.5) LEMMA. Let $K$ be a (smooth) simple closed curve in $M$ representing a class $\alpha \in H_{1}(M)$. For there to be an integral surgery along $K$ yielding a homology 3 -sphere, it is necessary and sufficient that $l_{M}(\alpha, \alpha) \equiv \pm 1 / r$. Further, if $l_{M}(\alpha, \alpha) \equiv$ $\pm 1 / r$ where $r$ is odd, there are knots $K_{1}$ and $K_{2}$ in $M$ representing $\alpha$ and integral surgeries along $K_{1}$ and $K_{2}$ such that the associated homology 3-spheres have distinct $\mu$-invariants.

Now as $H_{*}(M)$ is presented by $(\mathbf{Z}, L)$, there is an $\alpha \in H_{1}(M)$ such that $l_{M}(\alpha, \alpha)$ $\equiv-1 / r$. For each $u \in A(M)$, let $K_{u}$ be a closed curve representing $u \alpha$ and $A_{u}$ a framing of $K_{u}$ which gives a homology 3-sphere. 
If $V\left(A_{u}\right)$ is the 1-connected 4-manifold constructed in the discussion prior to the proof of Proposition (0.5), then

(i) $\partial V\left(A_{u}\right)=M$;

(ii) $\left(H_{2}\left(V\left(A_{u}\right)\right), \cdot\right) \cong(\mathbf{Z}, L)$.

(When $|r| \leq 2$, care must be taken in the choice of integral surgery so as to ensure (ii).) It can also be checked that if $\eta \in H_{2}\left(V\left(A_{u}\right), M\right) \cong \mathbf{Z}$ is a generator, then $\partial \eta= \pm u \alpha$. Using this observation we can construct isometries $\Lambda(u):(\mathbf{Z}, L) \rightarrow$ $\left(H_{2}\left(V\left(A_{u}\right)\right), \cdot\right)$ for which $\partial\left(\Lambda(u) \cdot \Lambda(1)^{-1}\right)_{1}=u$. But then, from the action of $A(M)$ on $B_{L}^{t}(M)(\mathrm{V} .3)$ we see

$$
c_{L}\left(\left[V\left(A_{u}\right)\right]\right)=u c_{L}\left(\left[V\left(A_{1}\right)\right]\right) .
$$

As $u$ was arbitrary, $c_{L}^{t}$ is onto. This together with Theorem (0.2) implies that $c_{L}^{t}: \mathcal{V}_{L}(M) \rightarrow B_{L}^{t}$ is bijective when $r$ is even.

Finally, when $r$ is odd, use Lemma (5.5) with identity (V.1) to deduce the bijectivity of $C_{L}^{t}: \mathcal{V}_{L}(M) \rightarrow B_{L}^{t}(M) \times \mathbf{Z} / 2$.

(5.6) REMARKS. (i) Suppose $M$ is the lens space $L(r, s)$. It is well known that

$$
H_{+}^{t}(L(r, s))= \begin{cases}\{ \pm 1\}, & s^{2} \not \equiv 1(\bmod r), \\ \{ \pm 1, \pm s\}, & s^{2} \equiv 1(\bmod r)\end{cases}
$$

(see Chapter $\mathrm{V}$ of $[\mathbf{C}]$ for instance). Thus

$$
V_{L}(M) \cong B_{L}^{t}(M) \cong \begin{cases}A^{t}(M) /\{ \pm 1\}, & s^{2} \not \equiv 1(\bmod r) \\ A^{t}(M) /\{ \pm 1, \pm s\}, & s^{2} \equiv 1(\bmod r)\end{cases}
$$

where $A^{t}(M)=\left\{u \mid u^{2} \equiv 1\right\} \subseteq \mathbf{Z} / r$.

(ii) When $r$ is even, it is natural to ask how random $\Delta: \mathcal{V}_{L}(M) \rightarrow \mathbf{Z} / 2$ is. By (V.1), $\Delta\left(V\left(A_{u}\right)\right) \equiv \mu\left(\Sigma\left(A_{u}\right)\right)$, and arguing as in [Fu] it is possible to show that $\mu\left(\Sigma\left(A_{u_{1}}\right)\right) \equiv \mu\left(\Sigma\left(A_{u_{2}}\right)\right)$ as long as $u_{1} u_{2} \equiv 1(\bmod 2 r)$. This though is the only restriction as enough examples may be constructed using the results in [Tu].

\section{BIBLIOGRAPHY}

$[\mathbf{A}, \mathbf{S}]$ M. Atiyah and I. Singer, Index of elliptic operators. III, Ann. of Math. (2) 87 (1968), $546-604$.

[B] S. Boyer, Shake-slice knots, Ph.D. Thesis, Cornell University, 1983.

[C] M. M. Cohen, A course in simple homotopy theory, Graduate Texts in Math., vol. 10, Springer-Verlag, Berlin and New York, 1970.

[Fr] M. Freedman, The topology of 4-manifolds, J. Differential Geom. 17 (1982), 357-453.

[Fu] S. Fukuhara, On an invariant of homology lens spaces, J. Math. Soc. Japan 36 (1984), 259-277.

[G,L] C. Gordon and R. Litherland, On the signature of a link, Invent. Math. 47 (1978), 53-69.

[Ka] S. Kaplan, Constructing framed 4-manifolds with given almost framed boundaries, Trans. Amer. Math. Soc. 254 (1979), 237-263.

[K] M. Kervaire, Relative characteristic classes, Amer. J. Math. 79 (1957), 517-558.

[Mi] J. Milnor, Spin structures on manifolds, Enseign. Math. 9 (1963), 198-203.

[Mo] J. Morgan, Letter to R. Kirby, 1975.

[Q] F. Quinn, Ends of maps. III, Dimensions 4 and 5, J. Differential Geom. 17 (1982), 503-521.

[Rlf] D. Rolfsen, Knots and links, Publish or Perish, Berkeley, Calif., 1976.

[S] L. Siebenmann, Topological manifolds, Actes du Congrès International des Mathématiciens (Nice, 1970), Tome 2, Gauthier-Villars, Paris, 1971, pp. 133-163.

[Ta] L. Taylor, Relative Rochlin invariants, Topology Appl. 18 (1984), 259-280.

[Tu] V. G. Turaev, Cohomology rings, linking forms and invariants of spin structures of three-dimensional manifolds, Math. USSR Sb. 48 (1984), 65-79. 
[V] P. Vogel, Simply-connected 4-manifolds, Sem. Notes 1, Aarhus Univ., Aarhus, 1982, pp. 116-119.

[W1] C. T. C. Wall, On simply-connected 4-manifolds, J. London Math. Soc. 39 (1964), 141149.

[W2] _ Q Quadratic forms on finite groups, and related topics, Topology 2 (1964), 281-298.

[Wu] W. T. Wu, Classes caractéristiques et i-carrés dans une variété, C. R. Acad. Sci. Paris Vie Académique, 230 (1950), 508-511.

Department of Mathematics, University of Toronto, Toronto, Ontario, CANADA M5S 1A1 